\title{
Application of Interval Neutrosophic Power Hamy Mean Operators in MAGDM
}

\author{
Peide LIU $^{*}$, Qaisar KHAN ${ }^{2}$, Tahir MAHMOOD ${ }^{2}$ \\ ${ }^{1}$ School of Management Science and Engineering, \\ Shandong University of Finance and Economics, Jinan Shandong 250014, China \\ ${ }^{2}$ Department of Mathematics and Statistic, International Islamic University, Islamabad, Pakistan \\ e-mail:peide.liu@gmail.com,qaisarkhan421@gmail.com,tahirbakhat@iiu.edu.pk
}

Received: August 2018; accepted: January 2019

\begin{abstract}
The Hamy mean (HM) operator, as a convenient mathematical aggregation tool, can deal with the interrelationship among multiple input parameters, and the power average (PA) operator can relieve the influence of awkward assessment values in the decision consequences. The interval neutrosophic sets (INSs) are a more powerful mathematical tool to handle insufficient, indeterminate and vague information that exists in real life problems. Yet, in some complicated decision-making situations, we require to consider the correlation between multi-input arguments and remove the influence of awkward data at the same time. To deal with such situations, in this paper, we combine the conventional HM operator to the traditional PA operator in interval neutrosophic settings and present two novel interval neutrosophic aggregation operators, that is, the interval neutrosophic power Hamy mean (INPHM) operator and the weighted interval neutrosophic power Hamy mean (WINPHM) operators. Then, some preferable properties of the developed aggregation operators are discussed. Moreover, based on these developed aggregation operators, we propose a new method for multiple attribute group decision making (MAGDM) under the INSs. Lastly, some examples are given to show the effectiveness of the developed method by comparing it with other existing methods.
\end{abstract}

Key words: interval neutrosophic sets, Hamy mean operators, power average operators, MAGDM.

\section{Introduction}

In our day-to-day life, MAGDM or multi-attribute decision making (MADM) problems are very usual, and they have attracted many researchers' concentrations. MAGDM is a procedure of ranking alternatives and selecting the most preferable alternative from a possible set of alternatives supervised by a group of decision makers (DMs) based on the evaluations of prominent and discarding attributes, quantitative and qualitative (Cabrerizo et al., 2017). In conventional MAGDM, all decision data are known exactly or given in crisp values. Because of the complication of decision making, it is hard for decision makers (DMs) to define information in exact numbers. In MADM or MAGDM problems, in order to define the fuzzy properties of distinct attributes in a better way, Zadeh (1965) developed the concept of fuzzy sets (FSs). In FSs there was only truth-membership degree

\footnotetext{
* Corresponding author.
} 
(TMD) and the falsity-membership degree (FMD) couldn't be defined. FSs were further extended by Atanassov (1986), and the concept of intuitionistic FSs (IFSs) was developed, which could solve this flaw very effectively. Because IFSs contained both TMD and FMD, they were extensively used to manifest the attribute values of the MADM and MAGDM problems since they were developed. However, FS and IFS can only deal with incomplete information, but cannot deal with vague and inconsistent information which occurs frequently in belief system. Therefore, in order to deal with such situation, Smarandache $(1998,1999)$ initially developed the concept of neutrosophic set (NS) by including an unconventional indeterminacy-membership degree (IMD) on the basis of IFS which means that the DMs explained their perception on an object by the use of TMD, IMD and FMD. However, because the concept of NSs was based on philosophical point of view and it contained the subsets of non-standard unit interval, it was complicated to use in real life and engineering problems. So in order to use NSs more easily, some researchers proposed various subclasses of NSs such as single valued neutrosophic sets (SVNSs) (Wang et al., 2010), interval neutrosophic sets (INSs) (Wang et al., 2005; Zhang et al., 2014), simplified neutrosophic sets (SNSs) (Peng et al., 2016; Ye, 2014), and so on. Further, Garg (2016) developed improved score function for NSs. Peng et al. (2014), Zhang et al. (2016), respectively, developed some improved operational laws, outranking relations for SNSs, INS and applied them to MADM. Bausys et al. (2015) extended conventional COPRAS method to deal with SVN information. Some other traditional methods such as MULTIMOORA (Stanujkic et al., 2017), WASPAS (Nie et al., 2017; Zavadskas et al., 2015), MAMVA (Zavadskas et al., 2017) and TODIM (Ji et al., 2018a) were extended to neutrosophic environment and applied in various areas. Recently, Feng et al. (2018) developed DEMATEL and ELECTRE III to deal with IN information. Huang et al. (2017) proposed VIKOR method for INSs and applied them to MAGDM problem. Several studies have been conducted for MAGDM problems and developed different models: Dong et al. $(2016,2018)$ proposed self management mechanism for non-cooperative behaviour in large scale group consensus reaching process to decrease the assessments values of decision makers with non-cooperative behaviour and investigated how to recognize and handle a series of non-cooperative behaviours in GDM consensus reaching procedures from different perspectives. Capuano et al. (2018) introduced a model which indicates the impact navigated between the development of experts' opinions and its convergence properties. Urena et al. (2019) proposed a mechanism to generate and propagate trust and reputation in social networks. Morente-Molinera et al. (2019) proposed a novel method which is capable of extracting collective knowledge of users' opinions and to express it in fuzzy ontology.

As one of the necessary tools for MAGDM or MADM, the information aggregation operators (AOs) have gained much more concentration from researchers and a lot of research achievements were made. The main goal of the AOs is to aggregate a sequence of input arguments into one (Xu, 2007; Xu and Yager, 2006). In general, AOs can include some particular functions. For example, PA operator, initially proposed by Yager (2001), which can eliminate the effect of awkward assessment values given by DMs by their own personal preferences. PA operators were further extended by many authors such as Xu 
(2011) who extended the PA operator to handle the information of IFS (IFPA), He et al. (2013) proposed the generalized PA operators for interval-valued IFS and applied them to MAGDM, Zhang et al. (2015) proposed Frank IFPA operators, Liu and Liu (2014), Liu and Tang (2016) proposed intuitionistic trapezoidal fuzzy power generalized AOs and power generalized AOs for INSs and applied them to MADM. Some AOs can only consider interrelationship between the input arguments such as Bonferroni mean (BM), Heronian mean, Maclaurin symmetric mean (MSM) operators. All these AOs were further extended by many researchers, such as Xu and Yager (2011) who proposed BM operator for IFS (IFBM), Yu (2015) introduced triangular Atanassov IFBM operator and applied it to supplier selection, Liu and Wang (2014) presented the concept of SVN normalized weighted BM operators, Ji et al. (2018b) proposed BM operator for INNs. Similar to BM, Heronian mean was also extended to deal with various types of information (Li et al., 2016; Liu and Chen, 2017; Yu and Wu, 2012). To take combined advantage of PA, BM and Heronian mean operators, some hybrid structure such as combining PA operator with BM operator, and PA operator with HM operator is also proposed (He et al., 2015a; Liu, 2017; He et al., 2015b; Liu and Li, 2017). All the above AOs can only reflect the influence of awkward data or consider the interrelationship between two input arguments or both at the same time but cannot consider the interrelationship among multi-input arguments. The MSM proposed by Maclaurin (1729) has the property that it can consider the interrelationship among the multi-input arguments. In recent years some researchers extended MSM operator to deal with various environment (Liu and Gao, 2018; Liu and Zhang, 2018; Qin and Liu, 2014; Wei and Lu, 2018; Wu et al., 2018). Liu and You (2017) extended Muirhead operators, which can also consider the interrelationship among the multi-input argument, to INS information and applied them to solve MADM problems. Recently, Liu et al. (2018) proposed the concept of power MSM for interval-valued IFSs and applied it in MAGDM, which can relieve the influence of the awkward data and can consider the interrelationship among multiple arguments at the same time. Obviously, they can only deal with interval-valued IFSs.

Hamy mean (HM) operator was first proposed by Hara et al. (1998), which has the property that it can consider the interrelation among multiple parameters by modifying the parameters values and it is a generalization of MSM and Muirhead operators. After that, HM operator was further extended by Qin (2017) to deal with interval type-2 fuzzy information. Liu and You (2018), Wu et al. (2018) further extended HM operators to deal with linguistic neutrosophic and 2-tuple linguistic neutrosophic information respectively. From the existing literature, there is no such AO to deal with the information of INSs, which has the capacity that it can diminish the influence of the awkward data and can consider the interrelationship among multiple arguments at the same time. Therefore, it is necessary to develop some new AOs by combining the ordinary PA operator with $\mathrm{HM}$ operator to deal with the information of INSs. These new AOs have four advantages. Firstly, they are better to deal with uncertain information by defining TMD, IMD and FMD from interval neutrosophic numbers (INNs). Secondly, they can relieve the influence of the awkward data given by biased DMs. Thirdly, they can consider the interrelationship among multiple arguments. Lastly, they are more flexible than the other AOs by modifying the parameter values. Hence, in this article, we will attain the following aims: 
1. Develop interval neutrosophic power HM (INPHM) operators and weighted INPHM operator.

2. Discuss properties and specific cases of these proposed AOs.

3. Propose an MAGDM approach based on the proposed AOs.

4. Express the effectiveness and practicality of the proposed approach.

To do so, the rest of this article is organized as follows. In Section 2, we initiated some basic ideas of INSs, PA operators, HM operators, consisting definitions, operational rules, distance measures, properties, score and accuracy functions. In Section 3, we propose INPHM operators by combining PA operator and HM operator, and also propose it in weighted form. Further we discuss some properties and special cases of the proposed AOs. In Section 4, we develop a MAGDM approach based on these AOs. In Section 5, we solve two numerical examples to show the validity and advantages of the proposed approach by comparing it with other existing methods.

\section{Preliminaries}

In this section, we briefly review some basic concepts about INSs, HM operators and PA operators.

\subsection{INSs and their operations}

Definition 1. (See Smarandache, 1998, 1999.) Let $\Theta$ be a space of points (objects), with a non-specific component in $\Theta$ expressed by $\kappa$. A NS $\widetilde{N E}$ in $\Theta$ is expressed by

$$
\widetilde{N E}=\left\{\left\langle\kappa, \xi_{\widetilde{N E}}(\kappa), \psi_{\widetilde{N E}}(\kappa), \zeta_{\widetilde{N E}}(\kappa)\right\rangle \mid \kappa \in \Theta\right\}
$$

where $\xi_{\widetilde{N E}}(\kappa), \psi_{\widetilde{N E}}(\kappa)$ and $\zeta_{\widetilde{N E}}(\kappa)$, respectively, express the TMD, IMD and FMD of the element $\kappa \in \Theta$ to the set $\widetilde{N E}$. For every point $\kappa \in \Theta$, we have $\xi_{\widetilde{N E}}(\kappa), \psi_{\widetilde{N E}}(\kappa), \zeta_{\widetilde{N E}}(\kappa) \in$ ] $0^{-}, 1^{+}\left[\right.$, and $0^{-} \leqslant \xi_{\widetilde{N E}}(\kappa)+\psi_{\widetilde{N E}}(\kappa)+\zeta_{\widetilde{N E}}(\kappa) \leqslant 3^{+}$.

The NS was predominantly presented from a philosophical point of view, and is difficult to apply in real life or engineering problems due to the containment of non-standard intervals. Then, Wang et al. (2010) presented a subclass of NS by changing the non-standard unit interval to standard unit interval, and named it SVNS. The definition of SVNS is given below:

Definition 2. (See Wang et al., 2010.) Let $\Theta$ be a space of points (objects), with a common component in $\Theta$ denoted by $\eta$. A SVNS $\widetilde{S N}$ in $\Theta$ is expressed by,

$$
\widetilde{S N}=\left\{\left\langle\eta, \xi_{\widetilde{S N}}(\eta), \psi_{\widetilde{S N}}(\eta), \zeta_{\widetilde{S N}}(\eta)\right\rangle \mid \eta \in \Theta\right\},
$$

where $\xi_{\widetilde{S N}}(\eta), \psi_{\widetilde{S N}}(\eta)$ and $\zeta_{\widetilde{S N}}(\eta)$, respectively, denote the TMD, IMD and FMD of the element $\eta \in \Theta$ to the set $\widetilde{S N}$. For each point $\eta \in \Theta$, we have $\xi_{\widetilde{S N}}(\eta), \psi_{\widetilde{S N}}(\eta), \zeta_{\widetilde{S N}}(\eta) \in$ $[0,1]$, and $0 \leqslant \xi_{\widetilde{S N}}(\eta)+\psi_{\widetilde{S N}}(\eta)+\zeta_{\widetilde{S N}}(\eta) \leqslant 3$. 
In order to define more complex information, Wang et al. (2005) further presented the concept of INS, shown as follows:

Definition 3. (See Wang et al., 2005.) Let $\Theta$ be a space of points (objects), with a common component in $\Theta$ denoted by $u$. A INS $\Re$ in $\Theta$ is expressed by,

$$
\bar{M}=\left\{\left\langle u,\left[\xi_{\bar{M}}^{L}(u), \xi_{\bar{M}}^{U}(u)\right],\left[\psi_{\bar{M}}^{L}(u), \psi_{\bar{M}}^{U}(u)\right],\left[\zeta_{\bar{M}}^{L}(u), \zeta_{\bar{M}}^{U}(u)\right]\right\rangle \mid u \in \Theta\right\},
$$

where $\left[\xi_{\bar{M}}^{L}(u), \xi_{\bar{M}}^{U}(u)\right],\left[\psi_{\bar{M}}^{L}(u), \psi_{\bar{M}}^{U}(u)\right]$ and $\left[\zeta_{\bar{M}}^{L}(u), \zeta_{\bar{M}}^{U}(u)\right]$, respectively, express the interval TMD (ITMD), interval IMD (IIMD) and interval FMD (IFMD) of the element $u \in \Theta$ to the set $\bar{M}$. For every point $u \in \Theta$, we have $\left[\xi_{\bar{M}}^{L}(u), \xi_{\bar{M}}^{U}(u)\right],\left[\psi_{\bar{M}}^{L}(u), \psi_{\bar{M}}^{U}(u)\right]$, $\left[\zeta_{\bar{M}}^{L}(u), \zeta_{\bar{M}}^{U}(u)\right] \subseteq[0,1]$ and $0 \leqslant \xi_{\Re}^{U}(u)+\psi_{\Re}^{U}(u)+\zeta_{\Re}^{U}(u) \leqslant 3$.

For computational simplicity, to express an element $u$ in an INS, we can use $\Re=$ $\left\langle\left[\xi^{L}, \xi^{U}\right],\left[\psi^{L}, \psi^{U}\right],\left[\zeta^{L}, \zeta^{U}\right]\right\rangle$, and the element $u$ is called an interval neutrosophic number (INN). Where $\left[\xi^{L}, \xi^{U}\right] \subseteq[0,1],\left[\psi^{L}, \psi^{U}\right] \subseteq[0,1],\left[\zeta^{L}, \zeta^{U}\right] \subseteq[0,1]$ and $0 \leqslant$ $\xi^{U}+\psi^{U}+\zeta^{U} \leqslant 3$.

Definition 4. (See Zhang et al., 2014.) Let $\Re_{1}=\left\langle\left[\xi_{1}^{L}, \xi_{1}^{U}\right],\left[\psi_{1}^{L}, \psi_{1}^{U}\right],\left[\zeta_{1}^{L}, \zeta_{1}^{U}\right]\right\rangle$ and $\Re_{2}=\left\langle\left[\xi_{2}^{L}, \xi_{2}^{U}\right],\left[\psi_{2}^{L}, \psi_{2}^{U}\right],\left[\zeta_{2}^{L}, \zeta_{2}^{U}\right]\right\rangle$ be any two INNs, and $\delta>0$. Then, the operational laws of INNs can be defined as follows:

(1) $\Re_{1} \oplus \Re_{2}=\left\langle\left[\xi_{1}^{L}+\xi_{2}^{L}-\xi_{1}^{L} \xi_{2}^{L}, \xi_{1}^{U}+\xi_{2}^{U}-\xi_{1}^{U} \xi_{2}^{U}\right],\left[\psi_{1}^{L} \psi_{2}^{L}, \psi_{1}^{U} \psi_{2}^{U}\right]\right.$,

$$
\left.\left[\zeta_{1}^{L} \zeta_{2}^{L}, \zeta_{1}^{U} \zeta_{2}^{U}\right]\right\rangle
$$

(2) $\Re_{1} \otimes \Re_{2}=\left\langle\left[\xi_{1}^{L} \xi_{2}^{L}, \xi_{1}^{U} \xi_{2}^{U}\right],\left[\psi_{1}^{L}+\psi_{2}^{L}-\psi_{1}^{L} \psi_{2}^{L}, \psi_{1}^{U}+\psi_{2}^{U}-\psi_{1}^{U} \psi_{2}^{U}\right]\right.$,

$$
\left.\left[\zeta_{1}^{L}+\zeta_{2}^{L}-\zeta_{1}^{L} \zeta_{2}^{L}, \zeta_{1}^{U}+\zeta_{2}^{U}-\zeta_{1}^{U} \zeta_{2}^{U}\right]\right)
$$

(3) $\Re_{1}^{\delta}=\left\langle\left[\left(\xi_{1}^{L}\right)^{\delta},\left(\xi_{1}^{U}\right)^{\delta}\right],\left[1-\left(1-\psi_{1}^{L}\right)^{\delta}, 1-\left(1-\psi_{1}^{U}\right)^{\delta}\right]\right.$,

$$
\left.\left[1-\left(1-\zeta_{1}^{L}\right)^{\delta}, 1-\left(1-\zeta_{1}^{U}\right)^{\delta}\right]\right) \text {; }
$$

$$
\text { (4) } \delta \Re_{1}=\left\langle\left[1-\left(1-\xi_{1}^{L}\right)^{\delta}, 1-\left(1-\xi_{1}^{U}\right)^{\delta}\right],\left[\left(\psi_{1}^{L}\right)^{\delta},\left(\psi_{1}^{U}\right)^{\delta}\right],\left[\left(\zeta_{1}^{L}\right)^{\delta},\left(\zeta_{1}^{U}\right)^{\delta}\right]\right\rangle \text {. }
$$

Definition 5. (See Liu and You, 2017.) Let $\Re=\left\langle\left[\xi^{L}, \xi^{U}\right],\left[\psi^{L}, \psi^{U}\right],\left[\zeta^{L}, \zeta^{U}\right]\right\rangle$ be an INN. Then the score function $\widetilde{S C}(\Re)$ and accuracy function $\widetilde{A C}(\Re)$ can be defined as follows:

(1) $\widetilde{S C}(\Re)=\frac{\xi^{L}+\xi^{U}}{2}+1-\frac{\psi^{L}+\psi^{U}}{2}+1-\frac{\zeta^{L}+\zeta^{U}}{2}$;

(2) $\widetilde{A C}(\Re)=\frac{\xi^{L}+\xi^{U}}{2}+\frac{\zeta^{L}+\zeta^{U}}{2}$.

For comparing two INNs, the comparison rules were defined by Liu and You (2017), which can be stated as follows. 
Definition 6. (See Liu and You, 2017.) Let $\Re_{1}=\left\langle\left[\xi_{1}^{L}, \xi_{1}^{U}\right],\left[\psi_{1}^{L}, \psi_{1}^{U}\right],\left[\zeta_{1}^{L}, \zeta_{1}^{U}\right]\right\rangle$ and $\Re_{2}=\left\langle\left[\xi_{2}^{L}, \xi_{2}^{U}\right],\left[\psi_{2}^{L}, \psi_{2}^{U}\right],\left[\zeta_{2}^{L}, \zeta_{2}^{U}\right]\right\rangle$ be any two INNs. Then, we have

(1) If $\widetilde{S C}\left(\Re_{1}\right)>\widetilde{S C}\left(\Re_{2}\right)$, then $\Re_{1}$ is better than $\Re_{2}$, and denoted by $\Re_{1}>\Re_{2}$;

(2) If $\widetilde{S C}\left(\Re_{1}\right)=\widetilde{S C}\left(\Re_{2}\right)$, and $\widetilde{A C}\left(\Re_{1}\right)>\widetilde{A C}\left(\Re_{2}\right)$, then $\Re_{1}$ is better than $\Re_{2}$, and denoted by $\Re_{1}>\Re_{2}$;

(3) If $\widetilde{S C}\left(\Re_{1}\right)=\widetilde{S C}\left(\Re_{2}\right)$, and $\widetilde{A C}\left(\Re_{1}\right)=\widetilde{A C}\left(\Re_{2}\right)$, then $\Re_{1}$ is equal to $\Re_{2}$, and denoted by $\Re_{1}=\Re_{2}$.

Definition 7. (See Liu and Tang, 2016.) Let $\Re_{1}=\left\langle\left[\xi_{1}^{L}, \xi_{1}^{U}\right],\left[\psi_{1}^{L}, \psi_{1}^{U}\right],\left[\zeta_{1}^{L}, \zeta_{1}^{U}\right]\right\rangle$ and $\Re_{2}=\left\langle\left[\xi_{2}^{L}, \xi_{2}^{U}\right],\left[\psi_{2}^{L}, \psi_{2}^{U}\right],\left[\zeta_{2}^{L}, \zeta_{2}^{U}\right]\right\rangle$ be any two INNs. Then the normalized Hamming distance between $\Re_{1}$ and $\Re_{2}$ is defined as follows:

$$
\begin{aligned}
\widetilde{D S}\left(\Re_{1}, \Re_{2}\right)= & \frac{1}{6}\left(\left|\xi_{1}^{L}-\xi_{2}^{L}\right|+\left|\xi_{1}^{U}-\xi_{2}^{U}\right|+\left|\psi_{1}^{L}-\psi_{2}^{L}\right|+\left|\psi_{1}^{U}-\psi_{2}^{U}\right|+\left|\zeta_{1}^{L}-\zeta_{2}^{L}\right|\right. \\
& \left.+\left|\zeta_{1}^{U}-\zeta_{2}^{U}\right|\right) .
\end{aligned}
$$

\subsection{The HM Operator}

Definition 8. (See Hara et al., 1998.) The HM operator is described as follows:

$$
H M^{(k)}\left(\aleph_{1}, \aleph_{2}, \ldots, \aleph_{z}\right)=\frac{\sum_{1 \leqslant i_{1}<i_{2}<\cdots<i_{k} \leqslant z}\left(\prod_{j=1}^{z} \aleph_{i_{j}}\right)^{\frac{1}{k}}}{C_{z}^{k}},
$$

where $k(1,2, \ldots, z)$ is a parameter and $i_{1}, i_{2}, \ldots, i_{k}$ are $k$ integer values taken from the set of $\{1,2, \ldots, z\}$ of $z$ integer values, $C_{z}^{k}$ expresses the binomial co-efficient and $C_{z}^{k}=$ $\frac{z !}{k !(z-k) !}$.

The HM operator has the following properties, which are described below:

(1) When $\aleph_{i}=\aleph(i=1,2, \ldots, z)$, then $H M^{(k)}\left(\aleph_{1}, \aleph_{2}, \ldots, \aleph_{z}\right)=\aleph ;$

(2) When $\aleph_{i} \leqslant \Im_{i}(i=1,2, \ldots, z)$, then $H M^{(k)}\left(\aleph_{1}, \aleph_{2}, \ldots, \aleph_{z}\right) \leqslant H M^{(k)}\left(\Im_{1}, \Im_{2}\right.$, $\left.\ldots, \mathfrak{\Im}_{z}\right)$

(3) $\min _{i} \aleph_{i} \leqslant H M^{(k)}\left(\aleph_{1}, \aleph_{2}, \ldots, \aleph_{z}\right) \leqslant \max \aleph_{i}(i=1,2, \ldots, z)$.

The HM operator has two specific cases, which are defined below:

(1) When $k=1, H M^{1}\left(\aleph_{1}, \aleph_{2}, \ldots, \aleph_{z}\right)=\frac{1}{k} \sum_{i=1}^{z} \aleph_{i}$, the HM operator degenerates into arithmetic mean operator.

(2) When $k=z, H M^{(z)}\left(\aleph_{1}, \aleph_{2}, \ldots, \aleph_{z}\right)=\left(\prod_{i=1}^{z} \aleph_{i}\right)^{\frac{1}{k}}$, the HM operator degenerates into geometric mean operator.

\subsection{The PA Operator}

Definition 9. (See Yager, 2001.) Let $\left\{\aleph_{1}, \aleph_{2}, \ldots, \aleph_{z}\right\}$ be a set of positive real numbers. A $P A$ operator is described as follows:

$$
P A\left(\aleph_{1}, \aleph_{2}, \ldots, \aleph_{z}\right)=\frac{\sum_{i=1}^{z}\left(1+T\left(\aleph_{i}\right)\right) \aleph_{i}}{\sum_{i=1}^{z}\left(1+T\left(\aleph_{i}\right)\right)},
$$


where $T\left(\aleph_{i}\right)=\sum_{\substack{i=1 \\ j \neq i}}^{z} \operatorname{Supp}\left(\aleph_{i}, \aleph_{j}\right)$, and $\operatorname{Supp}\left(\aleph_{i}, \aleph_{j}\right)$ is the support degree for $\aleph_{i}$ from $\aleph_{j}$ satisfying the following axioms:

(1) $\operatorname{Supp}\left(\aleph_{1}, \aleph_{j}\right) \in[0,1]$

(2) $\operatorname{Supp}\left(\aleph_{i}, \aleph_{j}\right)=\operatorname{Supp}\left(\aleph_{j}, \aleph_{i}\right)$;

(3) $\operatorname{Supp}\left(\aleph_{i}, \aleph_{j}\right) \geqslant \operatorname{Supp}\left(\aleph_{p}, \aleph_{q}\right)$, if $\left|\left(\aleph_{i}, \aleph_{j}\right)\right|<\left|\left(\aleph_{p}, \aleph_{q}\right)\right|$.

\section{Interval Neutrosophic Power Hamy Mean Aggregation Operators}

Definition 10. Let $\Re_{i}=\left\langle\left[\xi_{i}^{L}, \xi_{i}^{U}\right],\left[\psi_{i}^{L}, \psi_{i}^{U}\right],\left[\zeta_{i}^{L}, \zeta_{i}^{U}\right]\right\rangle(i=1,2, \ldots, m)$ be a group of INNs, and the parameter $k=1,2, \ldots, m$. Then an interval neutrosophic power HM aggregation operator is a function INPHM : $\Theta^{m} \rightarrow \Theta$ defined as follows.

$$
\operatorname{INPHM}^{(k)}\left(\Re_{1}, \Re_{2}, \ldots, \Re_{m}\right)=\frac{\sum_{1 \leqslant i_{1}<i_{2}<\cdots<i_{k} \leqslant m}\left(\prod_{j=1}^{k} \frac{m\left(1+T\left(\Re_{i_{j}}\right)\right) \Re_{i_{j}}}{\sum_{z=1}^{m}\left(1+T\left(\Re_{z}\right)\right)}\right)^{\frac{1}{k}}}{\left(\begin{array}{c}
m \\
k
\end{array}\right)}
$$

where $\Theta$ is the set of all INNs, and $\Xi_{z}=\frac{\left(1+T\left(\Re_{z}\right)\right)}{\sum_{z=1}^{n}\left(1+T\left(\Re_{z}\right)\right)}$, and $\sum_{z=1}^{n} \Xi_{k}=1 . T\left(\Re_{j}\right)=$ $\sum_{\substack{z=1 \\ z \neq j}}^{n} \operatorname{Supp}\left(\Re_{z}, \Re_{j}\right)$ is the support degree for $\Re_{z}$ from $\Re_{j}$, which satisfies the following properties:

(1) $\operatorname{Supp}\left(\Re_{z}, \Re_{j}\right) \in[0,1]$,

(2) $\operatorname{Supp}\left(\Re_{z}, \Re_{j}\right)=\operatorname{Supp}\left(\Re_{j}, \Re_{z}\right)$,

(3) if $\widetilde{D}\left(\Re_{z}, \Re_{j}\right) \leqslant \widetilde{D}\left(\Re_{x}, \Re_{y}\right)$, then $\operatorname{Supp}\left(\Re_{z}, \Re_{j}\right) \geqslant \operatorname{Supp}\left(\Re_{x}, \Re_{y}\right)$, where $\widetilde{d}\left(\Re_{z}, \Re_{j}\right)$ represent the distance measure between any two INNs defined in Definition 7. $\left(i_{1}, i_{2}, \ldots, i_{k}\right)$ traverse all the $k$-tuple combinations of $(1,2, \ldots, m)$. The denominator $\left(\begin{array}{l}n \\ k\end{array}\right)$ in the above equation (13) represents the binomial coefficient $\frac{m !}{k !(m-k) !}$ and $m$ is the balancing coefficient.

In order to write equation (13) in a simple form, we can define

$$
\Xi_{z}=\frac{\left(1+T\left(\Re_{z}\right)\right)}{\sum_{z=1}^{n}\left(1+T\left(\Re_{z}\right)\right)}
$$

then we call $\left(\Xi_{1}, \Xi_{2}, \ldots, \Xi_{n}\right)$ the power weight vector. Therefore, equation (13) can be written in a simplified form as follows:

$$
\operatorname{INPHM}^{(k)}\left(\Re_{1}, \Re_{2}, \ldots, \Re_{m}\right)=\frac{\sum_{1 \leqslant i_{1}<i_{2}<\cdots<i_{k} \leqslant m}\left(\prod_{j=1}^{k} m \Xi_{i_{j}} \Re_{i_{j}}\right)^{\frac{1}{k}}}{\left(\begin{array}{c}
m \\
k
\end{array}\right)} .
$$

Theorem 1. Let $\mathfrak{R}_{i}=\left\langle\left[\xi_{i}^{L}, \xi_{i}^{U}\right],\left[\psi_{i}^{L}, \psi_{i}^{U}\right],\left[\zeta_{i}^{L}, \zeta_{i}^{U}\right]\right\rangle(i=1,2, \ldots, m)$ be a group of INNs, and the parameter $k=1,2, \ldots, m$. Then the value aggregated utilizing equation 
(15) is still an INN, and

$$
\begin{aligned}
& \operatorname{INPHM}^{(k)}\left(\Re_{1}, \Re_{2}, \ldots, \Re_{m}\right) \\
& =\left\langle\left[ 1-\left(\prod_{1 \leqslant i_{1}<i_{2}<\cdots<i_{k} \leqslant m}\left(1-\left(\prod_{j=1}^{k}\left(1-\left(1-\xi_{i_{j}}^{L}\right)^{m \Xi_{i_{j}}}\right)\right)^{\frac{1}{k}}\right)\right)^{\frac{1}{\left(\begin{array}{c}
n \\
k
\end{array}\right)}}\right.\right. \\
& \left.1-\left(\prod_{1 \leqslant i_{1}<i_{2}<\cdots<i_{k} \leqslant m}\left(1-\left(\prod_{j=1}^{k}\left(1-\left(1-\xi_{i_{j}}^{U}\right)^{m \Xi_{i_{j}}}\right)\right)^{\frac{1}{k}}\right)\right)^{\frac{1}{\left(\begin{array}{c}
n \\
k
\end{array}\right)}}\right], \\
& {\left[\left(\prod_{1 \leqslant i_{1}<i_{2}<\cdots<i_{k} \leqslant m}\left(1-\left(\prod_{j=1}^{k}\left(1-\left(\psi_{i_{j}}^{L}\right)^{m \Xi_{i_{j}}}\right)\right)^{\frac{1}{k}}\right)\right)^{\frac{1}{\left(\begin{array}{c}
n \\
k
\end{array}\right)}},\right.} \\
& \left.\left(\prod_{1 \leqslant i_{1}<i_{2}<\cdots<i_{k} \leqslant m}\left(1-\left(\prod_{j=1}^{k}\left(1-\left(\psi_{i_{j}}^{U}\right)^{m \Xi_{i_{j}}}\right)\right)^{\frac{1}{k}}\right)\right)^{\frac{1}{\left(\begin{array}{c}
n \\
k
\end{array}\right)}}\right], \\
& {\left[\left(\prod_{1 \leqslant i_{1}<i_{2}<\cdots<i_{k} \leqslant m}\left(1-\left(\prod_{j=1}^{k}\left(1-\left(\zeta_{i_{j}}^{L}\right)^{m \Xi_{i_{j}}}\right)\right)^{\frac{1}{k}}\right)\right)^{\frac{1}{\left(\begin{array}{c}
n \\
k
\end{array}\right)}}\right.} \\
& \left.\left.\left(\prod_{1 \leqslant i_{1}<i_{2}<\cdots<i_{k} \leqslant m}\left(1-\left(\prod_{j=1}^{k}\left(1-\left(\zeta_{i_{j}}^{L}\right)^{m \Xi_{i_{j}}}\right)\right)^{\frac{1}{k}}\right)\right)^{\frac{1}{\left(\begin{array}{c}
n \\
k
\end{array}\right)}}\right]\right) .
\end{aligned}
$$

Proof. Based on the operational rules for INNs, we have

$$
\begin{aligned}
m \Xi_{i_{j}} \Re_{i_{j}}= & \left\langle\left[1-\left(1-\xi_{i_{j}}^{L}\right)^{m \Xi_{i_{j}}}, 1-\left(1-\xi_{i_{j}}^{U}\right)^{m \Xi_{i_{j}}}\right],\left[\left(\psi_{i_{j}}^{L}\right)^{m \Xi_{i_{j}}},\left(\psi_{i_{j}}^{U}\right)^{m \Xi_{i_{j}}}\right],\right. \\
& {\left.\left[\left(\zeta_{i_{j}}^{L}\right)^{m \Xi_{i_{j}}},\left(\zeta_{i_{j}}^{U}\right)^{m \Xi_{i_{j}}}\right]\right\rangle, }
\end{aligned}
$$

and,

$$
\begin{aligned}
\prod_{j=1}^{k} m \Xi_{i_{j}} \Re_{i_{j}}= & \left\langle\left[\prod_{j=1}^{k}\left(1-\left(1-\xi_{i_{j}}^{L}\right)^{m \Xi_{i_{j}}}\right), \prod_{j=1}^{k}\left(1-\left(1-\xi_{i_{j}}^{U}\right)^{m \Xi_{i_{j}}}\right)\right],\right. \\
& {\left[1-\prod_{j=1}^{k}\left(1-\left(\psi_{i_{j}}^{L}\right)^{m \Xi_{i_{j}}}\right), 1-\prod_{j=1}^{k}\left(1-\left(\psi_{i_{j}}^{U}\right)^{m \Xi_{i_{j}}}\right)\right], } \\
& {\left.\left[1-\prod_{j=1}^{k}\left(1-\left(\zeta_{i_{j}}^{L}\right)^{m \Xi_{i_{j}}}\right), 1-\prod_{j=1}^{k}\left(1-\left(\zeta_{i_{j}}^{U}\right)^{m \Xi_{i_{j}}}\right)\right]\right\rangle }
\end{aligned}
$$


So,

$$
\begin{aligned}
\left(\prod_{j=1}^{k} m \Xi_{i_{j}} \Re_{i_{j}}\right)^{\frac{1}{k}} & \left\langle\left[\left(\prod_{j=1}^{k}\left(1-\left(1-\xi_{i_{j}}^{L}\right)^{m \Xi_{i_{j}}}\right)\right)^{\frac{1}{k}},\left(\prod_{j=1}^{k}\left(1-\left(1-\xi_{i_{j}}^{U}\right)^{m \Xi_{i_{j}}}\right)\right)^{\frac{1}{k}}\right]\right. \\
= & {\left[1-\left(\prod_{j=1}^{k}\left(1-\left(\psi_{i_{j}}^{L}\right)^{m \Xi_{i_{j}}}\right)\right)^{\frac{1}{k}}, 1-\left(\prod_{j=1}^{k}\left(1-\left(\psi_{i_{j}}^{U}\right)^{m \Xi_{i_{j}}}\right)\right)^{\frac{1}{k}}\right] } \\
& {\left.\left[1-\left(\prod_{j=1}^{k}\left(1-\left(\zeta_{i_{j}}^{L}\right)^{m \Xi_{i_{j}}}\right)\right)^{\frac{1}{k}}, 1-\left(\prod_{j=1}^{k}\left(1-\left(\zeta_{i_{j}}^{U}\right)^{m \Xi_{i_{j}}}\right)\right)^{\frac{1}{k}}\right]\right\rangle }
\end{aligned}
$$

Then,

$$
\begin{aligned}
& \sum_{1 \leqslant i_{1}<i_{2}<\cdots<i_{k} \leqslant m}\left(\prod_{j=1}^{k} m \Xi_{i_{j}} \Re_{i_{j}}\right)^{\frac{1}{k}} \\
& =\left\langle\left[ 1-\prod_{1 \leqslant i_{1}<i_{2}<\cdots<i_{k} \leqslant m}\left(1-\left(\prod_{j=1}^{k}\left(1-\left(1-\xi_{i_{j}}^{L}\right)^{m \Xi_{i_{j}}}\right)\right)^{\frac{1}{k}}\right),\right.\right. \\
& \left.1-\prod_{1 \leqslant i_{1}<i_{2}<\cdots<i_{k} \leqslant m}\left(1-\left(\prod_{j=1}^{k}\left(1-\left(1-\xi_{i_{j}}^{U}\right)^{m \Xi_{i_{j}}}\right)\right)^{\frac{1}{k}}\right)\right], \\
& {\left[\prod_{1 \leqslant i_{1}<i_{2}<\cdots<i_{k} \leqslant m}\left(1-\left(\prod_{j=1}^{k}\left(1-\left(\psi_{i_{j}}^{L}\right)^{m \Xi_{i_{j}}}\right)\right)^{\frac{1}{k}}\right),\right.} \\
& \left.\prod_{1 \leqslant i_{1}<i_{2}<\cdots<i_{k} \leqslant m}\left(1-\left(\prod_{j=1}^{k}\left(1-\left(\psi_{i_{j}}^{U}\right)^{m \Xi_{i_{j}}}\right)\right)^{\frac{1}{k}}\right)\right] \\
& {\left[\prod_{1 \leqslant i_{1}<i_{2}<\cdots<i_{k} \leqslant m}\left(1-\left(\prod_{j=1}^{k}\left(1-\left(\zeta_{i_{j}}^{L}\right)^{m \Xi_{i_{j}}}\right)\right)^{\frac{1}{k}}\right),\right.} \\
& \left.\left.\prod_{1 \leqslant i_{1}<i_{2}<\cdots<i_{k} \leqslant m}\left(1-\left(\prod_{j=1}^{k}\left(1-\left(\zeta_{i_{j}}^{L}\right)^{m \Xi_{i_{j}}}\right)\right)^{\frac{1}{k}}\right)\right]\right\rangle \text {. }
\end{aligned}
$$


Hence,

$$
\begin{aligned}
& \frac{\sum_{1 \leqslant i_{1}<i_{2}<\cdots<i_{k} \leqslant m}\left(\prod_{j=1}^{k} m \Xi_{i_{j}} \Re_{i_{j}}\right)^{\frac{1}{k}}}{\left(\begin{array}{l}
n \\
k
\end{array}\right)} \\
& =\left\langle\left[ 1-\left(\prod_{1 \leqslant i_{1}<i_{2}<\cdots<i_{k} \leqslant m}\left(1-\left(\prod_{j=1}^{k}\left(1-\left(1-\xi_{i_{j}}^{L}\right)^{m \Xi_{i_{j}}}\right)\right)^{\frac{1}{k}}\right)\right)^{\frac{1}{\left(l_{k}\right)}},\right.\right. \\
& \left.1-\left(\prod_{1 \leqslant i_{1}<i_{2}<\cdots<i_{k} \leqslant m}\left(1-\left(\prod_{j=1}^{k}\left(1-\left(1-\xi_{i_{j}}^{U}\right)^{m \Xi_{i_{j}}}\right)\right)^{\frac{1}{k}}\right)\right)^{\frac{1}{\left(k_{k}\right)}}\right], \\
& {\left[\left(\prod_{1 \leqslant i_{1}<i_{2}<\cdots<i_{k} \leqslant m}\left(1-\left(\prod_{j=1}^{k}\left(1-\left(\psi_{i_{j}}^{L}\right)^{m \Xi_{i_{j}}}\right)\right)^{\frac{1}{k}}\right)\right)^{\frac{1}{\left(k_{k}\right)}},\right.} \\
& \left.\left(\prod_{1 \leqslant i_{1}<i_{2}<\cdots<i_{k} \leqslant m}\left(1-\left(\prod_{j=1}^{k}\left(1-\left(\psi_{i_{j}}^{U}\right)^{m \Xi_{i_{j}}}\right)\right)^{\frac{1}{k}}\right)\right)^{\frac{1}{\left(l_{k}\right)}}\right], \\
& {\left[\left(\prod_{1 \leqslant i_{1}<i_{2}<\cdots<i_{k} \leqslant m}\left(1-\left(\prod_{j=1}^{k}\left(1-\left(\zeta_{i_{j}}^{L}\right)^{m \Xi_{i_{j}}}\right)\right)^{\frac{1}{k}}\right)\right)^{\frac{1}{\varphi_{k}^{k}}},\right.} \\
& \left.\left.\left(\prod_{1 \leqslant i_{1}<i_{2}<\cdots<i_{k} \leqslant m}\left(1-\left(\prod_{j=1}^{k}\left(1-\left(\zeta_{i_{j}}^{L}\right)^{m \Xi_{i_{j}}}\right)\right)^{\frac{1}{k}}\right)\right)^{\frac{1}{T_{k}}}\right]\right) .
\end{aligned}
$$

Therefore,

$$
\begin{aligned}
I^{N P H M} M^{(k)}\left(\Re_{1}, \Re_{2}, \ldots, \Re_{m}\right) & \left\langle\left[ 1-\left(\prod_{1 \leqslant i_{1}<i_{2}<\cdots<i_{k} \leqslant m}\left(1-\left(\prod_{j=1}^{k}\left(1-\left(1-\xi_{i_{j}}^{L}\right)^{m \Xi_{i_{j}}}\right)\right)^{\frac{1}{k}}\right)\right)^{\frac{1}{(k)}},\right.\right. \\
& \left.1-\left(\prod_{1 \leqslant i_{1}<i_{2}<\cdots<i_{k} \leqslant m}\left(1-\left(\prod_{j=1}^{k}\left(1-\left(1-\xi_{i_{j}}^{U}\right)^{m \Xi_{i_{j}}}\right)\right)^{\frac{1}{k}}\right)\right)^{\frac{1}{(k)}}\right], \\
& {\left[\left(\prod_{1 \leqslant i_{1}<i_{2}<\cdots<i_{k} \leqslant m}\left(1-\left(\prod_{j=1}^{k}\left(1-\left(\psi_{i_{j}}^{L}\right)^{m \Xi_{i_{j}}}\right)\right)^{\frac{1}{k}}\right)\right)^{\frac{1}{(k)}},\right.} \\
& \left.\left(\prod_{1 \leqslant i_{1}<i_{2}<\cdots<i_{k} \leqslant m}\left(1-\left(\prod_{j=1}^{k}\left(1-\left(\psi_{i_{j}}^{U}\right)^{m \Xi_{i_{j}}}\right)\right)^{\frac{1}{k}}\right)\right)^{\frac{1}{(k)}}\right],
\end{aligned}
$$




$$
\begin{aligned}
& {\left[\left(\prod_{1 \leqslant i_{1}<i_{2}<\cdots<i_{k} \leqslant m}\left(1-\left(\prod_{j=1}^{k}\left(1-\left(\zeta_{i_{j}}^{L}\right)^{m \Xi_{i_{j}}}\right)\right)^{\frac{1}{k}}\right)\right)^{\frac{1}{\left(\frac{1}{k}\right)}},\right.} \\
& \left.\left.\left(\prod_{1 \leqslant i_{1}<i_{2}<\cdots<i_{k} \leqslant m}\left(1-\left(\prod_{j=1}^{k}\left(1-\left(\zeta_{i_{j}}^{L}\right)^{m \Xi_{i_{j}}}\right)\right)^{\frac{1}{k}}\right)\right)^{\frac{1}{\left(\frac{1}{k}\right)}}\right]\right\rangle .
\end{aligned}
$$

Now, we shall discuss some basic properties of INPHM operator, which are stated below:

Theorem 2 (Idempotency). If all $\Re_{i}=\Re=\left\langle\left[\xi^{L}, \xi^{U}\right],\left[\psi^{L}, \psi^{U}\right],\left[\zeta^{L}, \zeta^{U}\right]\right\rangle$ for $(i=$ $1,2, \ldots, m)$, then

$$
\operatorname{INPHM}^{(k)}(\Re, \Re, \ldots, \Re)=\Re .
$$

Proof. Since all $\Re_{i}=\Re=\left\langle\left[\xi^{L}, \xi^{U}\right],\left[\psi^{L}, \psi^{U}\right],\left[\zeta^{L}, \zeta^{U}\right]\right\rangle$ for $(i=1,2, \ldots, m)$, then $m \Xi_{i_{j}}=\frac{m\left(1+T\left(\Re_{i_{j}}\right)\right)}{\sum_{z=1}^{m}\left(1+T\left(\Re_{z}\right)\right)}=1$.

So, according to Theorem 1, we have

$$
\begin{aligned}
& \operatorname{INPHM}^{(k)}(\Re, \Re, \ldots, \Re \\
& =\left\langle\left[ 1-\left(\prod_{1 \leqslant i_{1}<i_{2}<\cdots<i_{k} \leqslant m}\left(1-\left(\left(\xi^{L}\right)^{k}\right)^{\frac{1}{k}}\right)\right)^{\frac{1}{(k)} k},\right.\right.
\end{aligned}
$$

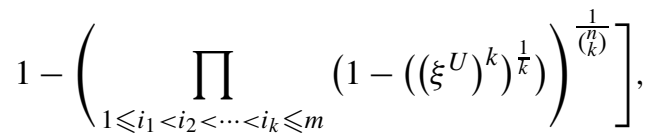

$$
\begin{aligned}
& {\left[\left(\prod_{1 \leqslant i_{1}<i_{2}<\cdots<i_{k} \leqslant m}\left(1-\left(\left(1-\psi^{L}\right)^{k}\right)^{\frac{1}{k}}\right)\right)^{\frac{1}{\left(\begin{array}{l}
n \\
k
\end{array}\right)}},\right.}
\end{aligned}
$$

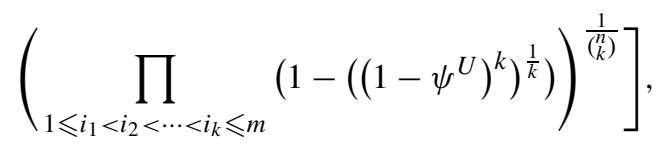

$$
\begin{aligned}
& {\left[\left(\prod_{1 \leqslant i_{1}<i_{2}<\cdots<i_{k} \leqslant m}\left(1-\left(\left(1-\zeta^{L}\right)^{k}\right)^{\frac{1}{k}}\right)\right)^{\frac{1}{\left(\frac{1}{k}\right)}},\right.} \\
& \left.\left.\left(\prod_{1 \leqslant i_{1}<i_{2}<\cdots<i_{k} \leqslant m}\left(1-\left(\left(1-\zeta^{U}\right)^{k}\right)^{\frac{1}{k}}\right)\right)^{\frac{1}{(n)}}\right]\right),
\end{aligned}
$$




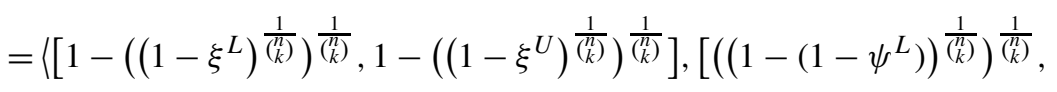

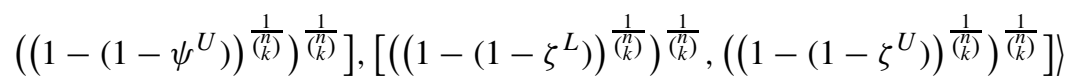

$$
\begin{aligned}
& =\left\langle\left[1-\left(1-\xi^{L}\right), 1-\left(1-\xi^{U}\right)\right],\left[\left(1-\left(1-\psi^{L}\right)\right),\left(1-\left(1-\psi^{U}\right)\right)\right],\right. \\
& \left.\left[\left(1-\left(1-\zeta^{L}\right)\right),\left(1-\left(1-\zeta^{U}\right)\right)\right]\right\rangle=\left\langle\left[\xi^{L}, \xi^{U}\right],\left[\psi^{L}, \psi^{U}\right],\left[\zeta^{L}, \zeta^{U}\right]\right\rangle \text {. }
\end{aligned}
$$

Theorem 3 (Commutativity). Let $\Re_{i}(i=1,2, \ldots, n)$ be a group of INNs, and $\widetilde{\Re}_{i}$ be any permutation of $\mathfrak{R}_{i}$. Then

$$
\operatorname{INPHM}^{(k)}\left(\Re_{1}, \Re_{2}, \ldots, \Re_{m}\right)=\operatorname{INPHM}^{(k)}\left(\widetilde{\Re}_{1}, \widetilde{\Re}_{2}, \ldots, \widetilde{\Re}_{m}\right) .
$$

Proof. Since $\left(\widetilde{\Re}_{1}, \widetilde{\Re}_{2}, \ldots, \widetilde{\Re}_{m}\right)$ is any permutation of $\left(\Re_{1}, \Re_{2}, \ldots, \Re_{m}\right)$, therefore, according to Definition 10, it is obvious that

$$
\begin{aligned}
& \operatorname{INPHM}^{(k)}\left(\widetilde{\Re}_{1}, \widetilde{\Re}_{2}, \ldots, \widetilde{\Re}_{m}\right)=\frac{\sum_{1 \leqslant i_{1}<i_{2}<\cdots<i_{k} \leqslant m}\left(\prod_{j=1}^{k} \frac{m\left(1+T\left(\widetilde{\Re}_{i_{j}}\right)\right) \widetilde{\Re}_{i_{j}}}{\sum_{z=1}^{m}\left(1+T\left(\Re_{z}\right)\right)}\right)^{\frac{1}{k}}}{\left(\begin{array}{l}
m \\
k
\end{array}\right)} \\
& =\frac{\sum_{1 \leqslant i_{1}<i_{2}<\cdots<i_{k} \leqslant m}\left(\prod_{j=1}^{k} \frac{m\left(1+T\left(\Re_{i_{j}}\right)\right) \Re_{i_{j}}}{\sum_{z=1}^{m}\left(1+T\left(\Re_{z}\right)\right)}\right)^{\frac{1}{k}}}{\left(\begin{array}{c}
m \\
k
\end{array}\right)} \\
& =I N P H M^{(k)}\left(\Re_{1}, \Re_{2}, \ldots, \Re_{m}\right) \text {. }
\end{aligned}
$$

Theorem 4 (Boundedness). Let $\Re_{i}(i=1,2, \ldots, n)$ be a group of INNs, and $\Re^{-}=$ $\min \left(\Re_{1}, \Re_{2}, \ldots, \Re_{m}\right)=\left\langle\left[\xi^{L}, \xi^{U}\right],\left[\psi^{L}, \psi^{U}\right],\left[\zeta^{L}, \zeta^{U}\right]\right\rangle, \Re^{+}=\max \left(\Re_{1}, \Re_{2}, \ldots, \Re_{m}\right)=$ $\left\langle\left[\widetilde{\xi}^{L}, \widetilde{\xi}^{U}\right],\left[\widetilde{\psi}^{L}, \widetilde{\psi}^{U}\right],\left[\widetilde{\zeta}^{L}, \widetilde{\zeta}^{U}\right]\right\rangle$. Then, the INPHM operator lies:

$$
\Re^{-} \leqslant \operatorname{INPHM}\left(\Re_{1}, \Re_{2}, \ldots, \Re_{m}\right) \leqslant \Re^{+}
$$

Proof. Since

$$
\begin{aligned}
m \Xi_{i_{j}} \Re_{i_{j}}= & \left\langle\left[1-\left(1-\xi_{i_{j}}^{L}\right)^{m \Xi_{i_{j}}}, 1-\left(1-\xi_{i_{j}}^{U}\right)^{m \Xi_{i_{j}}}\right],\left[\left(\psi_{i_{j}}^{L}\right)^{m \Xi_{i_{j}}},\left(\psi_{i_{j}}^{U}\right)^{m \Xi_{i_{j}}}\right],\right. \\
& {\left.\left[\left(\zeta_{i_{j}}^{L}\right)^{m \Xi_{i_{j}}},\left(\zeta_{i_{j}}^{U}\right)^{m \Xi_{i_{j}}}\right]\right\rangle } \\
\geqslant & \left\langle\left[1-\left(1-\xi^{L}\right)^{m \Xi_{i_{j}}}, 1-\left(1-\xi^{U}\right)^{m \Xi_{i_{j}}}\right],\left[\left(\psi^{L}\right)^{m \Xi_{i_{j}}},\left(\psi \psi^{U}\right)^{m \Xi_{i_{j}}}\right],\right. \\
& {\left.\left[\left(\zeta^{L}\right)^{m \Xi_{i_{j}}},\left(\zeta^{U}\right)^{m \Xi_{i_{j}}}\right]\right\rangle }
\end{aligned}
$$

and 


$$
\begin{aligned}
\prod_{j=1}^{k} m \Xi_{i_{j}} \Re_{i_{j}}= & \left\langle\left[\prod_{j=1}^{k}\left(1-\left(1-\xi_{i_{j}}^{L}\right)^{m \Xi_{i_{j}}}\right), \prod_{j=1}^{k}\left(1-\left(1-\xi_{i_{j}}^{U}\right)^{m \Xi_{i_{j}}}\right)\right],\right. \\
& {\left[1-\prod_{j=1}^{k}\left(1-\left(\psi_{i_{j}}^{L}\right)^{m \Xi_{i_{j}}}\right), 1-\prod_{j=1}^{k}\left(1-\left(\psi_{i_{j}}^{U}\right)^{m \Xi_{i_{j}}}\right)\right], } \\
& {\left.\left[1-\prod_{j=1}^{k}\left(1-\left(\zeta_{i_{j}}^{L}\right)^{m \Xi_{i_{j}}}\right), 1-\prod_{j=1}^{k}\left(1-\left(\zeta_{i_{j}}^{U}\right)^{m \Xi_{i_{j}}}\right)\right]\right\rangle } \\
\geqslant & \left\langle\left[\prod_{j=1}^{k}\left(1-\left(1-\xi^{L}\right)^{m \Xi_{i_{j}}}\right), \prod_{j=1}^{k}\left(1-\left(1-\xi^{U}\right)^{m \Xi_{i_{j}}}\right)\right],\right. \\
& {\left[1-\prod_{j=1}^{k}\left(1-\left(\psi^{L}\right)^{m \Xi_{i_{j}}}\right), 1-\prod_{j=1}^{k}\left(1-\left(\psi^{U}\right)^{m \Xi_{i_{j}}}\right)\right], } \\
& {\left.\left[1-\prod_{j=1}^{k}\left(1-\left(\zeta^{L}\right)^{m \Xi_{i_{j}}}\right), 1-\prod_{j=1}^{k}\left(1-\left(\zeta^{U}\right)^{m \Xi_{i_{j}}}\right)\right]\right\rangle . }
\end{aligned}
$$

So,

$$
\begin{aligned}
\left(\prod_{j=1}^{k} m \Xi_{i_{j}} \Re_{i_{j}}\right)^{\frac{1}{k}} & \left\langle\left[\left(\prod_{j=1}^{k}\left(1-\left(1-\xi_{i_{j}}^{L}\right)^{m \Xi_{i_{j}}}\right)\right)^{\frac{1}{k}},\left(\prod_{j=1}^{k}\left(1-\left(1-\xi_{i_{j}}^{U}\right)^{m \Xi_{i_{j}}}\right)\right)^{\frac{1}{k}}\right],\right. \\
& {\left[1-\left(\prod_{j=1}^{k}\left(1-\left(\psi_{i_{j}}^{L}\right)^{m \Xi_{i_{j}}}\right)\right)^{\frac{1}{k}}, 1-\left(\prod_{j=1}^{k}\left(1-\left(\psi_{i_{j}}^{U}\right)^{m \Xi_{i_{j}}}\right)\right)^{\frac{1}{k}}\right], } \\
\geqslant & {\left.\left.\left.\left[1-\left(\prod_{j=1}^{k}\left(1-\left(\zeta_{i_{j}}^{L}\right)^{m \Xi_{i_{j}}}\right)\right)^{\frac{1}{k}}, 1-\left(\prod_{j=1}^{k}\left(1-\left(1-\xi^{L}\right)^{m \Xi_{i_{j}}}\right)\right)^{\frac{1}{k}},\left(\zeta_{i_{j}}^{U}\right)^{m \Xi_{i_{j}}}\right)\right)^{\frac{1}{k}}\right]\right\rangle } \\
& {\left[1-\left(\prod_{j=1}^{k}\left(1-\left(1-\xi^{L}\right)^{m \Xi_{i_{j}}}\right)\right)^{\frac{1}{k}}\right], } \\
& {\left.\left.\left[1-\left(\psi^{L}\right)^{m \Xi_{i_{j}}}\right)\right)^{\frac{1}{k}}, 1-\left(\prod_{j=1}^{k}\left(1-\left(\psi^{U}\right)^{m \Xi_{i_{j}}}\right)\right)^{\frac{1}{k}}\right], } \\
& {\left.\left[1-\left(\prod_{j=1}^{k}\left(1-\left(\zeta^{L}\right)^{m \Xi_{i_{j}}}\right)\right)^{\frac{1}{k}}, 1-\left(\prod_{j=1}^{k}\left(1-\left(\zeta^{U}\right)^{m \Xi_{i_{j}}}\right)\right)^{\frac{1}{k}}\right]\right\rangle . }
\end{aligned}
$$


Then

$$
\begin{aligned}
& \sum_{1 \leqslant i_{1}<i_{2}<\cdots<i_{k} \leqslant m}\left(\prod_{j=1}^{k} m \Xi_{i_{j}} \Re_{i_{j}}\right)^{\frac{1}{k}} \\
& =\left\langle\left[ 1-\prod_{1 \leqslant i_{1}<i_{2}<\cdots<i_{k} \leqslant m}\left(1-\left(\prod_{j=1}^{k}\left(1-\left(1-\xi_{i_{j}}^{L}\right)^{m \Xi_{i_{j}}}\right)\right)^{\frac{1}{k}}\right),\right.\right. \\
& \left.1-\prod_{1 \leqslant i_{1}<i_{2}<\cdots<i_{k} \leqslant m}\left(1-\left(\prod_{j=1}^{k}\left(1-\left(1-\xi_{i_{j}}^{U}\right)^{m \Xi_{i_{j}}}\right)\right)^{\frac{1}{k}}\right)\right], \\
& {\left[\prod_{1 \leqslant i_{1}<i_{2}<\cdots<i_{k} \leqslant m}\left(1-\left(\prod_{j=1}^{k}\left(1-\left(\psi_{i_{j}}^{L}\right)^{m \Xi_{i_{j}}}\right)\right)^{\frac{1}{k}}\right),\right.} \\
& \left.\prod_{1 \leqslant i_{1}<i_{2}<\cdots<i_{k} \leqslant m}\left(1-\left(\prod_{j=1}^{k}\left(1-\left(\psi_{i_{j}}^{U}\right)^{m \Xi_{i_{j}}}\right)\right)^{\frac{1}{k}}\right)\right], \\
& {\left[\prod_{1 \leqslant i_{1}<i_{2}<\cdots<i_{k} \leqslant m}\left(1-\left(\prod_{j=1}^{k}\left(1-\left(\zeta_{i_{j}}^{L}\right)^{m \Xi_{i_{j}}}\right)\right)^{\frac{1}{k}}\right),\right.} \\
& \left.\left.\prod_{1 \leqslant i_{1}<i_{2}<\cdots<i_{k} \leqslant m}\left(1-\left(\prod_{j=1}^{k}\left(1-\left(\zeta_{i_{j}}^{L}\right)^{m \Xi_{i_{j}}}\right)\right)^{\frac{1}{k}}\right)\right]\right\rangle \\
& \geqslant\left\langle\left[ 1-\prod_{1 \leqslant i_{1}<i_{2}<\cdots<i_{k} \leqslant m}\left(1-\left(\prod_{j=1}^{k}\left(1-\left(1-\xi^{L}\right)^{m \Xi_{i_{j}}}\right)\right)^{\frac{1}{k}}\right)\right.\right. \text {, } \\
& \left.1-\prod_{1 \leqslant i_{1}<i_{2}<\cdots<i_{k} \leqslant m}\left(1-\left(\prod_{j=1}^{k}\left(1-\left(1-\xi^{U}\right)^{m \Xi_{i_{j}}}\right)\right)^{\frac{1}{k}}\right)\right] \text {, } \\
& {\left[\prod_{1 \leqslant i_{1}<i_{2}<\cdots<i_{k} \leqslant m}\left(1-\left(\prod_{j=1}^{k}\left(1-\left(\psi^{L}\right)^{m \Xi_{i j}}\right)\right)^{\frac{1}{k}}\right),\right.} \\
& \left.\prod_{1 \leqslant i_{1}<i_{2}<\cdots<i_{k} \leqslant m}\left(1-\left(\prod_{j=1}^{k}\left(1-\left(\psi^{L}\right)^{m \Xi_{i_{j}}}\right)\right)^{\frac{1}{k}}\right)\right], \\
& {\left[\prod_{1 \leqslant i_{1}<i_{2}<\cdots<i_{k} \leqslant m}\left(1-\left(\prod_{j=1}^{k}\left(1-\left(\zeta^{L}\right)^{m \Xi_{i_{j}}}\right)\right)^{\frac{1}{k}}\right),\right.} \\
& \left.\left.\prod_{1 \leqslant i_{1}<i_{2}<\cdots<i_{k} \leqslant m}\left(1-\left(\prod_{j=1}^{k}\left(1-\left(\zeta^{U}\right)^{m \Xi_{i_{j}}}\right)\right)^{\frac{1}{k}}\right)\right]\right\rangle \text {. }
\end{aligned}
$$


Hence,

$$
\begin{aligned}
& \frac{\sum_{1 \leqslant i_{1}<i_{2}<\cdots<i_{k} \leqslant m}\left(\prod_{j=1}^{k} m \Xi_{i_{j}} \Re_{i_{j}}\right)^{\frac{1}{k}}}{\left(\begin{array}{l}
n \\
k
\end{array}\right)} \\
& =\left\langle\left[ 1-\left(\prod_{1 \leqslant i_{1}<i_{2}<\cdots<i_{k} \leqslant m}\left(1-\left(\prod_{j=1}^{k}\left(1-\left(1-\xi_{i_{j}}^{L}\right)^{m \Xi_{i_{j}}}\right)\right)^{\frac{1}{k}}\right)\right)^{\frac{1}{\left(k_{k}\right)}},\right.\right. \\
& \left.1-\left(\prod_{1 \leqslant i_{1}<i_{2}<\cdots<i_{k} \leqslant m}\left(1-\left(\prod_{j=1}^{k}\left(1-\left(1-\xi_{i_{j}}^{U}\right)^{m \Xi_{i_{j}}}\right)\right)^{\frac{1}{k}}\right)\right)^{\frac{1}{\left(k_{k}\right)}}\right], \\
& {\left[\left(\prod_{1 \leqslant i_{1}<i_{2}<\cdots<i_{k} \leqslant m}\left(1-\left(\prod_{j=1}^{k}\left(1-\left(\psi_{i_{j}}^{L}\right)^{m \Xi_{i_{j}}}\right)\right)^{\frac{1}{k}}\right)\right)^{\frac{1}{(k)}},\right.} \\
& \left.\left(\prod_{1 \leqslant i_{1}<i_{2}<\cdots<i_{k} \leqslant m}\left(1-\left(\prod_{j=1}^{k}\left(1-\left(\psi_{i_{j}}^{U}\right)^{m \Xi_{i_{j}}}\right)\right)^{\frac{1}{k}}\right)\right)^{\left.\frac{1}{k_{k}}\right)}\right], \\
& {\left[\left(\prod_{1 \leqslant i_{1}<i_{2}<\cdots<i_{k} \leqslant m}\left(1-\left(\prod_{j=1}^{k}\left(1-\left(\zeta_{i_{j}}^{L}\right)^{m \Xi_{i_{j}}}\right)\right)^{\frac{1}{k}}\right)\right)^{\frac{1}{(k)}},\right.} \\
& \left.\left.\left(\prod_{1 \leqslant i_{1}<i_{2}<\cdots<i_{k} \leqslant m}\left(1-\left(\prod_{j=1}^{k}\left(1-\left(\zeta_{i_{j}}^{L}\right)^{m \Xi_{i_{j}}}\right)\right)^{\frac{1}{k}}\right)\right)^{\frac{1}{(k)}}\right]\right\rangle \\
& \geqslant\left\langle\left[ 1-\left(\prod_{1 \leqslant i_{1}<i_{2}<\cdots<i_{k} \leqslant m}\left(1-\left(\prod_{j=1}^{k}\left(1-\left(1-\xi^{L}\right)^{m \Xi_{i_{j}}}\right)\right)^{\frac{1}{k}}\right)\right)^{\frac{1}{\left(k_{k}\right)}}\right.\right. \text {, } \\
& \left.1-\left(\prod_{1 \leqslant i_{1}<i_{2}<\cdots<i_{k} \leqslant m}\left(1-\left(\prod_{j=1}^{k}\left(1-\left(1-\xi^{U}\right)^{m \Xi_{i_{j}}}\right)\right)^{\frac{1}{k}}\right)\right)^{\frac{1}{k_{k}}}\right], \\
& {\left[\left(\prod_{1 \leqslant i_{1}<i_{2}<\cdots<i_{k} \leqslant m}\left(1-\left(\prod_{j=1}^{k}\left(1-\left(\psi^{L}\right)^{m \Xi_{i_{j}}}\right)\right)^{\frac{1}{k}}\right)\right)^{\frac{1}{\left(h_{k}\right)}},\right.} \\
& \left.\left(\prod_{1 \leqslant i_{1}<i_{2}<\cdots<i_{k} \leqslant m}\left(1-\left(\prod_{j=1}^{k}\left(1-\left(\psi^{U}\right)^{m \Xi_{i_{j}}}\right)\right)^{\frac{1}{k}}\right)\right)^{\frac{1}{\left(k_{k}\right)}}\right], \\
& {\left[\left(\prod_{1 \leqslant i_{1}<i_{2}<\cdots<i_{k} \leqslant m}\left(1-\left(\prod_{j=1}^{k}\left(1-\left(\zeta^{L}\right)^{m \Xi_{i_{j}}}\right)\right)^{\frac{1}{k}}\right)\right)^{\frac{1}{(k)}},\right.} \\
& \left.\left.\left(\prod_{1 \leqslant i_{1}<i_{2}<\cdots<i_{k} \leqslant m}\left(1-\left(\prod_{j=1}^{k}\left(1-\left(\zeta^{U}\right)^{m \Xi_{i_{j}}}\right)\right)^{\frac{1}{k}}\right)\right)^{\frac{1}{\left(⿳_{k}\right.}}\right]\right\rangle \text {. }
\end{aligned}
$$


Therefore,

$$
\begin{aligned}
& \operatorname{INNPHM}^{(k)}\left(\Re_{1}, \Re_{2}, \ldots, \Re_{m}\right) \\
& =\left\langle\left[ 1-\left(\prod_{1 \leqslant i_{1}<i_{2}<\cdots<i_{k} \leqslant m}\left(1-\left(\prod_{j=1}^{k}\left(1-\left(1-\xi_{i_{j}}^{L}\right)^{m \Xi_{i_{j}}}\right)\right)^{\frac{1}{k}}\right)\right)^{\frac{1}{\left(c_{k}^{k}\right)}},\right.\right. \\
& \left.1-\left(\prod_{1 \leqslant i_{1}<i_{2}<\cdots<i_{k} \leqslant m}\left(1-\left(\prod_{j=1}^{k}\left(1-\left(1-\xi_{i_{j}}^{U}\right)^{m \Xi_{i_{j}}}\right)\right)^{\frac{1}{k}}\right)\right)^{\frac{1}{(k)}}\right], \\
& {\left[\left(\prod_{1 \leqslant i_{1}<i_{2}<\cdots<i_{k} \leqslant m}\left(1-\left(\prod_{j=1}^{k}\left(1-\left(\psi_{i_{j}}^{L}\right)^{m \Xi_{i_{j}}}\right)\right)^{\frac{1}{k}}\right)\right)^{\frac{1}{\left(\begin{array}{l}
n \\
k
\end{array}\right)}},\right.} \\
& \left.\left(\prod_{1 \leqslant i_{1}<i_{2}<\cdots<i_{k} \leqslant m}\left(1-\left(\prod_{j=1}^{k}\left(1-\left(\psi_{i_{j}}^{U}\right)^{m \Xi_{i_{j}}}\right)\right)^{\frac{1}{k}}\right)\right)^{\frac{1}{(n)} k}\right], \\
& {\left[\left(\prod_{1 \leqslant i_{1}<i_{2}<\cdots<i_{k} \leqslant m}\left(1-\left(\prod_{j=1}^{k}\left(1-\left(\zeta_{i_{j}}^{L}\right)^{m \Xi_{i_{j}}}\right)\right)^{\frac{1}{k}}\right)\right)^{\left.\frac{1}{(n)}\right)},\right.} \\
& \left.\left.\left(\prod_{1 \leqslant i_{1}<i_{2}<\cdots<i_{k} \leqslant m}\left(1-\left(\prod_{j=1}^{k}\left(1-\left(\zeta_{i_{j}}^{L}\right)^{m \Xi_{i_{j}}}\right)\right)^{\frac{1}{k}}\right)\right)^{\frac{1}{(k)}}\right]\right\rangle \\
& \geqslant\left\langle\left[ 1-\left(\prod_{1 \leqslant i_{1}<i_{2}<\cdots<i_{k} \leqslant m}\left(1-\left(\prod_{j=1}^{k}\left(1-\left(1-\xi^{L}\right)^{m \Xi_{i_{j}}}\right)\right)^{\frac{1}{k}}\right)\right)^{\frac{1}{\left.\underline{T}_{k}^{k}\right)}},\right.\right. \\
& \left.1-\left(\prod_{1 \leqslant i_{1}<i_{2}<\cdots<i_{k} \leqslant m}\left(1-\left(\prod_{j=1}^{k}\left(1-\left(1-\xi^{U}\right)^{m \Xi_{i_{j}}}\right)\right)^{\frac{1}{k}}\right)\right)^{\frac{1}{(k)}}\right], \\
& {\left[\left(\prod_{1 \leqslant i_{1}<i_{2}<\cdots<i_{k} \leqslant m}\left(1-\left(\prod_{j=1}^{k}\left(1-\left(\psi^{L}\right)^{m \Xi_{i_{j}}}\right)\right)^{\frac{1}{k}}\right)\right)^{\frac{1}{\left(c_{k}\right)}},\right.} \\
& \left.\left(\prod_{1 \leqslant i_{1}<i_{2}<\cdots<i_{k} \leqslant m}\left(1-\left(\prod_{j=1}^{k}\left(1-\left(\psi^{U}\right)^{m \Xi_{i_{j}}}\right)\right)^{\frac{1}{k}}\right)\right)^{\frac{1}{(k)} k}\right] \text {, } \\
& {\left[\left(\prod_{1 \leqslant i_{1}<i_{2}<\cdots<i_{k} \leqslant m}\left(1-\left(\prod_{j=1}^{k}\left(1-\left(\zeta^{L}\right)^{m \Xi_{i_{j}}}\right)\right)^{\frac{1}{k}}\right)\right)^{\left.\frac{1}{(n)}\right)},\right.} \\
& \left.\left.\left(\prod_{1 \leqslant i_{1}<i_{2}<\cdots<i_{k} \leqslant m}\left(1-\left(\prod_{j=1}^{k}\left(1-\left(\zeta^{U}\right)^{m \Xi_{i_{j}}}\right)\right)^{\frac{1}{k}}\right)\right)^{\frac{1}{\left(\frac{1}{k}\right)}}\right]\right\rangle \\
& =\Re^{-} \text {. }
\end{aligned}
$$


Similarly, we can prove that $\operatorname{INPHM}\left(\Re_{1}, \Re_{2}, \ldots, \Re_{m}\right) \leqslant \Re^{+}$. Hence $\Re^{-} \leqslant \operatorname{INPHM}\left(\Re_{1}\right.$, $\left.\Re_{2}, \ldots, \Re_{m}\right) \leqslant \Re^{+}$.

In what follows, we shall discuss some special cases of INPHM operators with respect to the parameter $k$, which were stated below.

(1). When $k=1$, the INPHM operator in equation (16), will degenerate to the following form:

$$
\begin{aligned}
& \operatorname{INPHM}^{(1)}\left(\Re_{1}, \Re_{2}, \ldots, \Re_{m}\right) \\
& =\frac{\sum_{1 \leqslant i_{1} \leqslant m}\left(\prod_{j=1}^{1} \frac{m\left(1+T\left(\Re_{i_{j}}\right)\right) \Re_{i_{j}}}{\sum_{z=1}^{m}\left(1+T\left(\Re_{z}\right)\right)}\right)^{\frac{1}{1}}}{\left(\begin{array}{c}
m \\
1
\end{array}\right)}, \\
& =\left\langle\left[ 1-\left(\prod_{1 \leqslant i_{1}<\leqslant m}\left(1-\left(\prod_{j=1}^{1}\left(1-\left(1-\xi_{i_{j}}^{L}\right)^{m \Xi_{i_{j}}}\right)\right)^{\frac{1}{\mathrm{~T}}}\right)\right)^{\frac{1}{(1)}},\right.\right. \\
& \left.1-\left(\prod_{1 \leqslant i_{1} \leqslant m}\left(1-\left(\prod_{j=1}^{1}\left(1-\left(1-\xi_{i_{j}}^{U}\right)^{m \Xi_{i_{j}}}\right)\right)^{\frac{1}{1}}\right)\right)^{\frac{1}{\left(\begin{array}{c}
n \\
1
\end{array}\right)}}\right] \text {, } \\
& {\left[\left(\prod_{1 \leqslant i_{1} \leqslant m}\left(1-\left(\prod_{j=1}^{1}\left(1-\left(\psi_{i_{j}}^{L}\right)^{m \Xi_{i_{j}}}\right)\right)^{\frac{1}{\mathrm{~T}}}\right)\right)^{\frac{1}{\left(c_{1}^{n}\right)}},\right.} \\
& \left.\left(\prod_{1 \leqslant i_{1} \leqslant m}\left(1-\left(\prod_{j=1}^{1}\left(1-\left(\psi_{i_{j}}^{U}\right)^{m \Xi_{i_{j}}}\right)\right)^{\frac{1}{T}}\right)\right)^{\frac{1}{\left(\begin{array}{c}
n \\
1
\end{array}\right)}}\right] \text {, } \\
& {\left[\left(\prod_{1 \leqslant i_{1} \leqslant m}\left(1-\left(\prod_{j=1}^{k}\left(1-\left(\zeta_{i_{j}}^{L}\right)^{m \Xi_{i_{j}}}\right)\right)^{\frac{1}{1}}\right)\right)^{\frac{1}{\left(\begin{array}{c}
n \\
1
\end{array}\right)}}\right.} \\
& \left.\left.\left(\prod_{1 \leqslant i_{1}<\leqslant m}\left(1-\left(\prod_{j=1}^{1}\left(1-\left(\zeta_{i_{j}}^{L}\right)^{m \Xi_{i_{j}}}\right)\right)^{\frac{1}{\mathrm{~T}}}\right)\right)^{\frac{1}{\left(\begin{array}{c}
n \\
1
\end{array}\right)}}\right]\right\rangle, \\
& =\left\langle\left[1-\left(\prod_{i=1}^{m}\left(1-\xi_{i}^{L}\right)^{m \Xi_{i}}\right)^{\frac{1}{m}}, 1-\left(\prod_{i=1}^{m}\left(1-\xi_{i}^{U}\right)^{m \Xi_{i}}\right)^{\frac{1}{m}}\right]\right. \text {, } \\
& {\left[\left(\prod_{i=1}^{m}\left(\psi_{i}^{L}\right)^{m \Xi_{i}}\right)^{\frac{1}{m}},\left(\prod_{i=1}^{m}\left(\psi_{i}^{U}\right)^{m \Xi_{i}}\right)^{\frac{1}{m}}\right]} \\
& \left.\left[\left(\prod_{i=1}^{m}\left(\zeta_{i}^{L}\right)^{m \Xi_{i}}\right)^{\frac{1}{m}},\left(\prod_{i=1}^{m}\left(\zeta_{i}^{U}\right)^{m \Xi_{i}}\right)^{\frac{1}{m}}\right]\right\rangle \quad\left(\operatorname{let} i_{1}=i\right)
\end{aligned}
$$




$$
=\frac{1}{m} \sum_{i=1}^{m} m \Xi_{i} \Re_{i}=I N P A\left(\Re_{1}, \Re_{2}, \ldots, \Re_{m}\right),
$$

i.e. when $k=1$, the INPHM operator degenerates into power averaging operator proposed by Liu and Tang (2016).

(2). When $k=m$, then the INPHM operator degenerates into the following form:

$$
\begin{aligned}
& \operatorname{INPHM}^{(m)}\left(\Re_{1}, \Re_{2}, \ldots, \Re_{m}\right) \\
& =\frac{\sum_{1 \leqslant i_{1}<i_{2}<\cdots<i_{k} \leqslant m}\left(\prod_{j=1}^{m} \frac{m\left(1+T\left(\Re_{i_{j}}\right)\right) \Re_{i_{j}}}{\sum_{z=1}^{m}\left(1+T\left(\Re_{z}\right)\right)}\right)^{\frac{1}{m}}}{\left(\begin{array}{l}
m \\
m
\end{array}\right)}, \\
& =\left\langle\left[ 1-\left(\prod_{1 \leqslant i_{1}<i_{2}<\cdots<i_{k} \leqslant m}\left(1-\left(\prod_{j=1}^{m}\left(1-\left(1-\xi_{i_{j}}^{L}\right)^{m \Xi_{i_{j}}}\right)\right)^{\frac{1}{m}}\right)\right)^{\frac{1}{(m)}},\right.\right. \\
& \left.1-\left(\prod_{1 \leqslant i_{1}<i_{2}<\cdots<i_{k} \leqslant m}\left(1-\left(\prod_{j=1}^{m}\left(1-\left(1-\xi_{i_{j}}^{U}\right)^{m \Xi_{i_{j}}}\right)\right)^{\frac{1}{m}}\right)\right)^{\frac{1}{\left(\begin{array}{c}
n \\
m
\end{array}\right)}}\right] \\
& {\left[\left(\prod_{1 \leqslant i_{1}<i_{2}<\cdots<i_{k} \leqslant m}\left(1-\left(\prod_{j=1}^{m}\left(1-\left(\psi_{i_{j}}^{L}\right)^{m \Xi_{i_{j}}}\right)\right)^{\frac{1}{m}}\right)\right)^{\frac{1}{\left(\begin{array}{c}
n \\
m
\end{array}\right)}},\right.} \\
& \left.\left(\prod_{1 \leqslant i_{1}<i_{2}<\cdots<i_{k} \leqslant m}\left(1-\left(\prod_{j=1}^{m}\left(1-\left(\psi_{i_{j}}^{U}\right)^{m \Xi_{i_{j}}}\right)\right)^{\frac{1}{m}}\right)\right)^{\frac{1}{\left(\begin{array}{c}
n \\
m
\end{array}\right)}}\right], \\
& {\left[\left(\prod_{1 \leqslant i_{1}<i_{2}<\cdots<i_{k} \leqslant m}\left(1-\left(\prod_{j=1}^{m}\left(1-\left(\zeta_{i_{j}}^{L}\right)^{m \Xi_{i_{j}}}\right)\right)^{\frac{1}{m}}\right)\right)^{\frac{1}{(m)}},\right.} \\
& \left.\left.\left(\prod_{1 \leqslant i_{1}<i_{2}<\cdots<i_{k} \leqslant m}\left(1-\left(\prod_{j=1}^{m}\left(1-\left(\zeta_{i_{j}}^{L}\right)^{m \Xi_{i_{j}}}\right)\right)^{\frac{1}{m}}\right)\right)^{\frac{1}{\left(\begin{array}{c}
n \\
m
\end{array}\right)}}\right]\right\rangle \\
& =\left\langle\left[\left(\prod_{j=1}^{m}\left(1-\left(1-\xi_{i_{j}}^{L}\right)^{m \Xi_{i_{j}}}\right)\right)^{\frac{1}{m}},\left(\prod_{j=1}^{m}\left(1-\left(1-\xi_{i_{j}}^{U}\right)^{m \Xi_{i_{j}}}\right)\right)^{\frac{1}{m}}\right]\right. \text {, } \\
& {\left[1-\left(\prod_{j=1}^{m}\left(1-\left(\psi_{i_{j}}^{L}\right)^{m \Xi_{i_{j}}}\right)\right)^{\frac{1}{m}}, 1-\left(\prod_{j=1}^{m}\left(1-\left(\psi_{i_{j}}^{U}\right)^{m \Xi_{i_{j}}}\right)\right)^{\frac{1}{m}}\right],} \\
& \left.\left[1-\left(\prod_{j=1}^{m}\left(1-\left(\zeta_{i_{j}}^{L}\right)^{m \Xi_{i_{j}}}\right)\right)^{\frac{1}{m}}, 1-\left(\prod_{j=1}^{m}\left(1-\left(\zeta_{i_{j}}^{U}\right)^{m \Xi_{i_{j}}}\right)\right)^{\frac{1}{m}}\right]\right\rangle \text {. }
\end{aligned}
$$


Further, if we suppose that $\operatorname{Supp}\left(\Re_{i}, \Re_{j}\right)=\beta$ for all $i \neq j$, then $m \Xi_{i_{j}}=\frac{m\left(1+T\left(\Re_{i_{j}}\right)\right)}{\sum_{z=1}^{m}\left(1+T\left(\Re_{z}\right)\right)}$ $=1$, and equation (16) can further degenerate into the following form:

$$
\begin{aligned}
= & \left\langle\left[\left(\prod_{j=1}^{m}\left(\xi_{i_{j}}^{L}\right)\right)^{\frac{1}{m}},\left(\prod_{j=1}^{m} \xi_{i_{j}}^{U}\right)^{\frac{1}{m}}\right],\left[1-\left(\prod_{j=1}^{m}\left(1-\psi_{i_{j}}^{L}\right)\right)^{\frac{1}{m}}, 1-\left(\prod_{j=1}^{m}\left(1-\psi_{i_{j}}^{U}\right)\right)^{\frac{1}{m}}\right]\right. \\
& {\left.\left[1-\left(\prod_{j=1}^{m}\left(1-\zeta_{i_{j}}^{L}\right)\right)^{\frac{1}{m}}, 1-\left(\prod_{j=1}^{m}\left(1-\zeta_{i_{j}}^{U}\right)\right)^{\frac{1}{m}}\right]\right\rangle }
\end{aligned}
$$

That is, equation (16) degenerates into ING operator.

In the INPHM operator, we can notice that only the interrelation among inputs arguments and the power weight vector are taken into consideration, the weight vector of the aggregated arguments is ignored. However, in some situations, the importance degree of the attributes is an important factor in the aggregation process, especially, in MAGDM. So in order to overcome this deficiency, the weighted form of the INPHM operator is defined as follows.

Definition 11. Let $\Re_{i}=\left\langle\left[\xi_{i}^{L}, \xi_{i}^{U}\right],\left[\psi_{i}^{L}, \psi_{i}^{U}\right],\left[\zeta_{i}^{L}, \zeta_{i}^{U}\right]\right\rangle(i=1,2, \ldots, m)$ be a group of INNs, and the parameter $k=1,2, \ldots, m$. Then a weighted interval neutrosophic power HM operator is a function WINPHM : $\Theta^{m} \rightarrow \Theta$ defined as follows:

$$
\operatorname{WINPHM}^{(k)}\left(\Re_{1}, \Re_{2}, \ldots, \Re_{m}\right)=\frac{\sum_{1 \leqslant i_{1}<i_{2}<\cdots<i_{k} \leqslant m}\left(\prod_{j=1}^{k} m \Upsilon_{i_{j}} \Re_{i_{j}}\right)^{\frac{1}{k}}}{\left(\begin{array}{c}
m \\
k
\end{array}\right)}
$$

where $\Theta$ is the set of all INNs, and $\Upsilon_{i}=\frac{\varpi_{i}\left(1+T\left(\Re_{i}\right)\right)}{\sum_{z=1}^{n} \varpi_{i}\left(1+T\left(\Re_{z}\right)\right)}, T\left(\Re_{j}\right)=\sum_{\substack{z=1 \\ z \neq j}}^{n} \operatorname{Supp}\left(\Re_{z}, \Re_{j}\right)$ is the support degree for $\Re_{z}$ from $\Re_{j}$, which satisfies the following properties; (1) $\operatorname{Supp}\left(\Re_{z}, \Re_{j}\right) \in[0,1],(2) \operatorname{Supp}\left(\Re_{z}, \Re_{j}\right)=\operatorname{Supp}\left(\Re_{j}, \Re_{z}\right),(3) \widetilde{D}\left(\Re_{z}, \Re_{j}\right) \leqslant \widetilde{D}\left(\Re_{x}, \Re_{y}\right)$, then $\operatorname{Supp}\left(\Re_{z}, \Re_{j}\right) \geqslant \operatorname{Supp}\left(\Re_{x}, \Re_{y}\right)$, where $\widetilde{d}\left(\Re_{z}, \Re_{j}\right)$ represents the distance measure between any two INNs defined in Definition $7, \varpi=\left(\varpi_{1}, \varpi_{2}, \ldots, \varpi_{m}\right)^{T}$ is the weight vector of $\Re_{i}(i=1,2, \ldots, m)$ such that $\varpi_{i} \in[0,1]$ and $\sum_{i=1}^{m} \varpi_{i}=1\left(i_{1}, i_{2}, \ldots, i_{k}\right)$ traverse all the $k$-tuple combinations of $(1,2, \ldots, m)$. The denominator $\left(\begin{array}{c}m \\ k\end{array}\right)$ in the above equation (23) represents the binomial coefficient, $\frac{m !}{k !(m-k) !}$ and $m$ are the balancing coefficients.

Theorem 5. Let $\Re_{i}=\left\langle\left[\xi_{i}^{L}, \xi_{i}^{U}\right],\left[\psi_{i}^{L}, \psi_{i}^{U}\right],\left[\zeta_{i}^{L}, \zeta_{i}^{U}\right]\right\rangle(i=1,2, \ldots, m)$ be a group of INNs, and the parameter $k=1,2, \ldots, m$. Then, the value aggregated utilizing equation (23) is still an INN, and

$$
\begin{aligned}
& \operatorname{WINPHM}^{(k)}\left(\Re_{1}, \Re_{2}, \ldots, \Re_{m}\right) \\
& \quad=\left\langle\left[ 1-\left(\prod_{1 \leqslant i_{1}<i_{2}<\cdots<i_{k} \leqslant m}\left(1-\left(\prod_{j=1}^{k}\left(1-\left(1-\xi_{i_{j}}^{L}\right)^{m \varpi_{i_{j}}}\right)\right)^{\frac{1}{k}}\right)\right)^{\frac{1}{\left(\begin{array}{c}
n \\
k
\end{array}\right)}},\right.\right.
\end{aligned}
$$




$$
\begin{aligned}
& \left.1-\left(\prod_{1 \leqslant i_{1}<i_{2}<\cdots<i_{k} \leqslant m}\left(1-\left(\prod_{j=1}^{k}\left(1-\left(1-\xi_{i_{j}}^{U}\right)^{m \varpi_{i_{j}}}\right)\right)^{\frac{1}{k}}\right)\right)^{\frac{1}{\left(\begin{array}{c}
n \\
k
\end{array}\right)}}\right], \\
& {\left[\left(\prod_{1 \leqslant i_{1}<i_{2}<\cdots<i_{k} \leqslant m}\left(1-\left(\prod_{j=1}^{k}\left(1-\left(\psi_{i_{j}}^{L}\right)^{m \varpi_{i_{j}}}\right)\right)^{\frac{1}{k}}\right)\right)^{\frac{1}{\left(\begin{array}{c}
n \\
k
\end{array}\right)}},\right.} \\
& \left.\left(\prod_{1 \leqslant i_{1}<i_{2}<\cdots<i_{k} \leqslant m}\left(1-\left(\prod_{j=1}^{k}\left(1-\left(\psi_{i_{j}}^{U}\right)^{m \varpi_{i_{j}}}\right)\right)^{\frac{1}{k}}\right)\right)^{\frac{1}{\left(\begin{array}{c}
n \\
k
\end{array}\right)}}\right], \\
& {\left[\left(\prod_{1 \leqslant i_{1}<i_{2}<\cdots<i_{k} \leqslant m}\left(1-\left(\prod_{j=1}^{k}\left(1-\left(\zeta_{i_{j}}^{L}\right)^{m \varpi_{i_{j}}}\right)\right)^{\frac{1}{k}}\right)\right)^{\frac{1}{(n)}(k)},\right.} \\
& \left.\left.\left(\prod_{1 \leqslant i_{1}<i_{2}<\cdots<i_{k} \leqslant m}\left(1-\left(\prod_{j=1}^{k}\left(1-\left(\zeta_{i_{j}}^{L}\right)^{m \varpi_{i_{j}}}\right)\right)^{\frac{1}{k}}\right)\right)^{\frac{1}{(n)} k}\right]\right\rangle .
\end{aligned}
$$

Proof. Proof of this theorem is the same as of Theorem 1.

Theorem 6 (Idempotency). If all $\Re_{i}=\Re=\left\langle\left[\xi^{L}, \xi^{U}\right],\left[\psi^{L}, \psi^{U}\right],\left[\zeta^{L}, \zeta^{U}\right]\right\rangle$ for $(i=$ $1,2, \ldots, m)$, then

$$
\operatorname{WINPHM}^{(k)}(\mathfrak{R}, \Re, \ldots, \mathfrak{R})=\Re .
$$

Theorem 7 (Commutativity). Let $\Re_{i}(i=1,2, \ldots, n)$ be a group of INNs, and $\widetilde{\Re}_{i}$ be any permutation of $\Re_{i}$. Then,

$$
\operatorname{WINPHM}^{(k)}\left(\Re_{1}, \Re_{2}, \ldots, \Re_{m}\right)=\operatorname{WINPHM}^{(k)}\left(\widetilde{\Re}_{1}, \widetilde{\Re}_{2}, \ldots, \widetilde{\Re}_{m}\right) .
$$

Theorem 8 (Boundedness). Let $\Re_{i}(i=1,2, \ldots, n)$ be a group of INNs, and $\Re^{-}=$ $\min \left(\Re_{1}, \Re_{2}, \ldots, \Re_{m}\right)=\left\langle\left[\xi^{L}, \xi^{U}\right],\left[\psi^{L}, \psi^{U}\right],\left[\zeta^{L}, \zeta^{U}\right]\right\rangle, \Re^{+}=\max \left(\Re_{1}, \Re_{2}, \ldots, \Re_{m}\right)=$ $\left\langle\left[\widetilde{\xi}^{L}, \widetilde{\xi}^{U}\right],\left[\widetilde{\psi}^{L}, \widetilde{\psi}^{U}\right],\left[\widetilde{\zeta}^{L}, \widetilde{\zeta}^{U}\right]\right\rangle$. Then, the INPHM operator lies:

$$
\mathfrak{R}^{-} \leqslant \operatorname{WINPHM}\left(\mathfrak{R}_{1}, \mathfrak{R}_{2}, \ldots, \mathfrak{R}_{m}\right) \leqslant \mathfrak{R}^{+} .
$$

The proofs of the above Theorems are same as the proofs of the Theorems for INPHM operator, therefore, omitted here.

\section{MAGDM Approach Based on Developed WINPHM Operator}

In this part, we will utilize the developed WINPHM operator to deal with MAGDM problem with the data presented in the form of INNs. Let the set of $m$ alternatives be denoted by $M=\left\{M_{1}, M_{2}, \ldots, M_{m}\right\}$ and the group of $n$ attributes be denoted by $\Xi=$ 
$\left\{\Xi_{1}, \Xi_{2}, \ldots, \Xi_{n}\right\}$, the importance degree of $n$ attributes be $\Upsilon=\left(\Upsilon_{1}, \Upsilon_{2}, \ldots, \Upsilon_{n}\right)^{T}$, such that $\Upsilon_{j} \in[0,1], j=1,2, \ldots, n, \sum_{j=1}^{n} \Upsilon_{j}=1$. There is a set of $z$ experts expressed by $\Psi=\left\{\Psi_{1}, \Psi_{2}, \ldots, \Psi_{z}\right\}$ who are asked to provide the assessment information, and the importance degree of the experts is expressed by $\Omega=\left(\Omega_{1}, \Omega_{2}, \ldots, \Omega_{z}\right)^{T}$, such that $\Omega_{a} \in[0,1],(a=1,2, \ldots, z), \sum_{a=1}^{z} \Omega_{a}=1$. The expert $\Psi_{a}$ assesses every attribute $\Xi_{j}$ of every alternative $\mathrm{M}_{i}$ by the form of INN $\Re_{i j}^{a}=\left\langle\left[\xi_{i j}^{a L}, \xi_{i j}^{a U}\right],\left[\psi_{i j}^{a L}, \psi_{i j}^{a}\right],\left[\zeta_{i j}^{a L}, \zeta_{i j}^{a U}\right]\right\rangle$ $(i=1,2, \ldots, m ; 1,2, \ldots, n)$, then the decision matrices $\widetilde{D M}_{a}=\left(\Re_{i j}^{a}\right)(a=1,2, \ldots, z)$ are established. The subsequent purpose is to execute a ranking of all alternatives.

Then, in order to solve this problem, we will execute the following steps:

Step 1. Firstly, the given decision matrices $\widetilde{D M}_{a}=\left(\Re_{i j}^{a}\right)_{m \times n}$ should be transformed into standardized decision matrices $\widetilde{S D M}_{a}=\left(\Re_{i j}^{a}\right)_{m \times n}$. We change the cost-type attribute into benefit-type attribute using the following formula.

$$
\begin{aligned}
& \Re_{i j}^{a}=\left\{\begin{array}{l}
\Re_{i j}^{a}=\left\langle\left[\xi_{i j}^{a}{ }^{L}, \xi_{i j}^{a U}\right],\left[\psi_{i j}^{a}{ }^{L}, \psi_{i j}^{a} U\right.\right. \\
\left.\quad \text { for benefit-type attribute } \Xi_{j},\left[\zeta_{i j}^{a L}, \zeta_{i j}^{a U}\right]\right\rangle \\
\left(\Re_{i j}^{a}\right)^{c}=\left\langle\left[\zeta_{i j}^{a}{ }^{L}, \zeta_{i j}^{a}{ }^{a}\right],\left[1-\psi_{i j}^{a} U, 1-\psi_{i j}^{a}{ }^{L}\right],\left[\xi_{i j}^{a}{ }^{L}, \xi_{i j}^{a} U\right]\right\rangle \\
\quad \text { for cost-type attribute } \Xi_{j} .
\end{array}\right. \\
& i=1,2, \ldots, m, j=1,2, \ldots, n .
\end{aligned}
$$

Step 2. Determine the supports

$$
\operatorname{Supp}\left(\Re_{i j}^{c}, \Re_{i j}^{d}\right)=1-\widetilde{D S}\left(\Re_{i j}^{c}, \Re_{i j}^{d}\right), \quad c, d=1,2, \ldots, z,
$$

which fulfills the required axioms given in Definition 10 , and $\widetilde{D S}\left(\Re_{i j}^{c}, \Re_{i j}^{d}\right)$ represents the distance measure given in Definition 7.

Step 3. Determine the supports $T\left(\Re_{i j}^{c}\right)$ of the INN $\Re_{i j}^{c}$ by other $\Re_{i j}^{d}(d=1,2, \ldots, z$ and $c \neq d)$.

$$
\begin{aligned}
T\left(\Re_{i j}^{c}\right) & =\sum_{d=1 ; c \neq d}^{z} \Omega_{d} \operatorname{Supp}\left(\Re_{i j}^{c}, \Re_{i j}^{d}\right) ; \\
c, d & =1,2, \ldots, z ; i=1,2, \ldots, m, j=1,2, \ldots, n .
\end{aligned}
$$

Then use the importance degrees $\Omega_{c}(c=1,2, \ldots, z)$ of the DMs $\Psi_{a}(a=1,2, \ldots, z)$ to calculate the importance degrees

$$
\varpi_{i j}^{(c)}=\frac{\Omega_{c}\left(1+T\left(\Re_{i j}^{c}\right)\right)}{\sum_{d}^{z} \Omega_{d}\left(1+T\left(\Re_{i j}^{d}\right)\right)} ; \quad c=1,2, \ldots, z ; i=1,2, \ldots, m, j=1,2, \ldots, n,
$$

where $\varpi_{i j} \geqslant 0$ and $\sum_{c=1}^{z} \varpi_{i j}=1$. 
Step 4. Utilize the WINPHM operator expressed by equation (24)

$$
\begin{aligned}
& \Re_{i j}=\operatorname{WSVNPHM}^{(k)}\left(\Re_{i j}^{(1)}, \Re_{i j}^{(2)}, \ldots, \Re_{i j}^{(z)}\right) \\
& =\left\langle\left[ 1-\left(\prod_{1 \leqslant i_{1}<i_{2}<\cdots<i_{k} \leqslant z}\left(1-\left(\prod_{j=1}^{k}\left(1-\left(1-\xi_{i_{j}}^{L}\right)^{z \varpi_{i}}\right)\right)^{\frac{1}{k}}\right)\right)^{\frac{1}{\left(\begin{array}{l}
z \\
k
\end{array}\right)}},\right.\right. \\
& \left.1-\left(\prod_{1 \leqslant i_{1}<i_{2}<\cdots<i_{k} \leqslant z}\left(1-\left(\prod_{j=1}^{k}\left(1-\left(1-\xi_{i_{j}}^{U}\right)^{z \varpi_{i_{j}}}\right)\right)^{\frac{1}{k}}\right)\right)^{\frac{1}{\left(\frac{2}{k}\right)}}\right], \\
& {\left[\left(\prod_{1 \leqslant i_{1}<i_{2}<\cdots<i_{k} \leqslant z}\left(1-\left(\prod_{j=1}^{k}\left(1-\left(\psi_{i_{j}}^{L}\right)^{z \varpi_{i_{j}}}\right)\right)^{\frac{1}{k}}\right)\right)^{\frac{1}{\left(\begin{array}{l}
2 \\
k
\end{array}\right)}},\right.}
\end{aligned}
$$

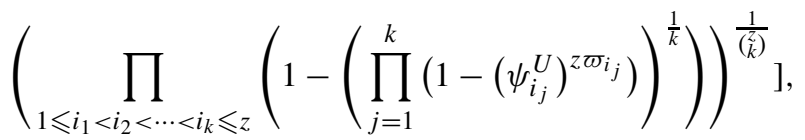

$$
\begin{aligned}
& {\left[\left(\prod_{1 \leqslant i_{1}<i_{2}<\cdots<i_{k} \leqslant z}\left(1-\left(\prod_{j=1}^{k}\left(1-\left(\zeta_{i_{j}}^{L}\right)^{z \varpi_{i_{j}}}\right)\right)^{\frac{1}{k}}\right)\right)^{\frac{1}{\left(\begin{array}{l}
2 \\
k
\end{array}\right)}},\right.} \\
& \left.\left.\left(\prod_{1 \leqslant i_{1}<i_{2}<\cdots<i_{k} \leqslant m}\left(1-\left(\prod_{j=1}^{k}\left(1-\left(\zeta_{i_{j}}^{L}\right)^{z \varpi_{i j}}\right)\right)^{\frac{1}{k}}\right)\right)^{\frac{1}{\left(\begin{array}{l}
2 \\
k
\end{array}\right)}}\right]\right\rangle
\end{aligned}
$$

to aggregate all the decision matrices $\widetilde{D M}_{a}=\left(\Re_{i j}^{a}\right)_{m \times n}(a=1,2, \ldots, z)$ given by the DMs into the comprehensive decision matrix $\widetilde{C D M}=\left(\Re_{i j}\right)_{m \times n}$.

Step 5. Determine the supports:

$$
\operatorname{Supp}\left(\Re_{i j}, \Re_{i q}\right)=1-\widetilde{D S}\left(\Re_{i j}, \Re_{i q}\right), \quad i=1,2, \ldots, m ; q=1,2, \ldots, n .
$$

which fulfils the required axioms given in Definition 10 , and $\widetilde{D S}\left(\Re_{i j}, \Re_{i q}\right)$ represents the distance measure given in Definition 7.

Step 6. Determine the supports $T\left(\Re_{i j}\right)$ of the INN $\Re_{i j}(i=1,2, \ldots, m ; j=1,2, \ldots, n)$ by the importance degrees $\Upsilon_{j}$ of the attributes $\Xi_{j}$ and the importance degrees $\phi_{i j}$ that are associated with the INN $\Re_{i j}$ by the importance degree $\Upsilon_{j}$ of the attributes $\Xi_{j}$.

$$
\begin{aligned}
& T\left(\Re_{i j}\right)=\sum_{q=1 ; q \neq j}^{z} \Upsilon_{j} \operatorname{Supp}\left(\Re_{i j}, \Re_{i q}\right), \quad i=1,2, \ldots, m, j, q=1,2, \ldots, n, \\
& \phi_{i j}=\frac{\Upsilon_{c}\left(1+T\left(\Re_{i j}\right)\right)}{\sum_{j=1}^{n} \Upsilon_{j}\left(1+T\left(\Re_{i j}\right)\right)} ; \quad i=1,2, \ldots, m, j=1,2, \ldots, n,
\end{aligned}
$$

where $\phi_{i j} \geqslant 0$ and $\sum_{c=1}^{z} \phi_{i j}=1$. 
Step 7. Utilize the WINPHM operator equation (24).

$$
\begin{aligned}
& \Re_{i}=\operatorname{WSVNPHM}^{(k)}\left(\Re_{i 1}, \Re_{i 2}, \ldots, \Re_{i n}\right) \\
& =\left\langle\left[ 1-\left(\prod_{1 \leqslant i_{1}<i_{2}<\cdots<i_{k} \leqslant n}\left(1-\left(\prod_{j=1}^{k}\left(1-\left(1-\xi_{i_{j}}^{L}\right)^{n \phi_{i_{j}}}\right)\right)^{\frac{1}{k}}\right)\right)^{\frac{1}{(k)} k},\right.\right. \\
& \left.1-\left(\prod_{1 \leqslant i_{1}<i_{2}<\cdots<i_{k} \leqslant n}\left(1-\left(\prod_{j=1}^{k}\left(1-\left(1-\xi_{i_{j}}^{U}\right)^{n \phi_{i_{j}}}\right)\right)^{\frac{1}{k}}\right)\right)^{\frac{1}{\left(\begin{array}{c}
n \\
k
\end{array}\right)}}\right], \\
& {\left[\left(\prod_{1 \leqslant i_{1}<i_{2}<\cdots<i_{k} \leqslant n}\left(1-\left(\prod_{j=1}^{k}\left(1-\left(\psi_{i_{j}}^{L}\right)^{n \phi_{i_{j}}}\right)\right)^{\frac{1}{k}}\right)\right)^{\frac{1}{\left(\frac{n}{k}\right)}},\right.} \\
& \left.\left(\prod_{1 \leqslant i_{1}<i_{2}<\cdots<i_{k} \leqslant n}\left(1-\left(\prod_{j=1}^{k}\left(1-\left(\psi_{i_{j}}^{U}\right)^{n \phi_{i_{j}}}\right)\right)^{\frac{1}{k}}\right)\right)^{\frac{1}{\left(\frac{n}{k}\right)}}\right], \\
& {\left[\left(\prod_{1 \leqslant i_{1}<i_{2}<\cdots<i_{k} \leqslant n}\left(1-\left(\prod_{j=1}^{k}\left(1-\left(\zeta_{i_{j}}^{L}\right)^{n \phi_{i_{j}}}\right)\right)^{\frac{1}{k}}\right)\right)^{\frac{1}{\left({ }_{k}^{n}\right)}},\right.} \\
& \left.\left.\left(\prod_{1 \leqslant i_{1}<i_{2}<\cdots<i_{k} \leqslant n}\left(1-\left(\prod_{j=1}^{k}\left(1-\left(\zeta_{i_{j}}^{L}\right)^{n \phi_{i_{j}}}\right)\right)^{\frac{1}{k}}\right)\right)^{\frac{1}{(n)}}\right]\right\rangle
\end{aligned}
$$

to get the comprehensive evaluation value.

Step 8. Determine the score and accuracy value of each INN $\Re_{i}(i=1,2, \ldots, n)$ using Definition 5.

Step 9. Rank all the alternatives and select the best one using Definition 6.

\section{Numerical Examples}

In this part, two numerical examples will be provided to show the application and advantages of proposed approach. The first example is about the selection of emerging technology enterprises (ETEs), see Huang et al. (2017).

\subsection{An Illustrative Example}

Let us assume that there are five ETEs represented by $\Theta_{i}(i=1,2,3,4,5)$, which are selected. These five ETEs are evaluated with respect to the following four attributes $\wp_{j}$ $(1,2,3,4)$, which are (1) $\wp_{1}$ : the employment formation, (2) $\wp_{2}$ : the progress of science and technology, (3) $\wp_{3}$ : technical improvement, (4) $\wp_{4}$ : the industrialization configuration. 
Table 1

The decision matrix $\widetilde{D M}_{1}$.

\begin{tabular}{lllll}
\hline & $\wp_{1}$ & $\wp_{2}$ & $\wp_{3}$ & $\wp_{4}$ \\
\hline$\Theta_{1}$ & $\langle[0.3,0.4],[0.6,0.7],[0.30 .5]\rangle$ & $\langle[0.4,0.5],[0.2,0.3],[0.1,0.2]\rangle$ & $\langle[0.1,0.2],[0.4,0.5],[0.1,0.2]\rangle$ & $\langle[0.3,0.4],[0.5,0.6],[0.2,0.3]\rangle$ \\
$\Theta_{2}$ & $\langle[0.5,0.7],[0.6,0.8],[0.2,0.4]\rangle$ & $\langle[0.5,0.6],[0.3,0.5],[0.2,0.3]\rangle$ & $\langle[0.5,0.7],[0.4,0.6],[0.2,0.3]\rangle$ & $\langle[0.6,0.7],[0.3,0.4],[0.2,0.3]\rangle$ \\
$\Theta_{3}$ & $\langle[0.4,0.5],[0.5,0.6],[0.2,0.3]\rangle$ & $\langle[0.3,0.4],[0.5,0.6],[0.1,0.2]\rangle$ & $\langle[0.3,0.4],[0.1,0.2],[0.2,0.3]\rangle$ & $\langle[0.4,0.5],[0.1,0.2],[0.3,0.4]\rangle$ \\
$\Theta_{4}$ & $\langle[0.6,0.7],[0.2,0.3],[0.1,0.2]\rangle$ & $\langle[0.4,0.5],[0.10 .2],[0.2,0.3]\rangle$ & $\langle[0.4,0.5],[0.2,0.3],[0.1,0.2]\rangle$ & $\langle[0.3,0.4],[0.4,0.5],[0.2,0.3]\rangle$ \\
$\Theta_{5}$ & $\langle[0.40 .5],[0.2,0.3],[0.2,0.3]\rangle$ & $\langle[0.2,0.3],[0.6,0.7],[0.2,0.3]\rangle$ & $\langle[0.5,0.6],[0.4,0.5],[0.2,0.3]\rangle$ & $\langle[0.3,0.4],[0.6,0.7],[0.3,0.4]\rangle$ \\
\hline
\end{tabular}

Table 2

The decision matrix $\widetilde{D M}_{2}$.

\begin{tabular}{lllll}
\hline & $\wp_{1}$ & $\wp_{2}$ & $\wp_{3}$ & $\wp_{4}$ \\
\hline$\Theta_{1}$ & $\langle[0.4,0.6],[0.5,0.7],[0.3,0.4]\rangle$ & $\langle[0.6,0.7],[0.5,0.6],[0.5,0.6]\rangle$ & $\langle[0.5,0.6],[0.4,0.5],[0.3,0.4]\rangle$ & $\langle[0.6,0.7],[0.4,0.5],[0.3,0.4]\rangle$ \\
$\Theta_{3}$ & $\langle[0.6,0.9],[0.4,0.5],[0.3,0.4]\rangle$ & $\langle[0.7,0.8],[0.6,0.7],[0.4,0.5]\rangle$ & $\langle[0.7,0.8],[0.3,0.4],[0.3,0.4]\rangle$ & $\langle[0.8,0.9],[0.4,0.5],[0.3,0.4]\rangle$ \\
$\Theta_{3}$ & $\langle[0.8,0.9],[0.8,0.9],[0.4,0.5]\rangle$ & $\langle[0.7,0.8],[0.5,0.6],[0.5,0.6]\rangle$ & $\langle[0.7,0.8],[0.1,0.2],[0.3,0.4]\rangle$ & $\langle[0.8,0.9],[0.5,0.6],[0.2,0.3]\rangle$ \\
$\Theta_{4}$ & $\langle[0.6,0.7],[0.3,0.4],[0.5,0.6]\rangle$ & $\langle[0.8,0.9],[0.5,0.6],[0.6,0.7]\rangle$ & $\langle[0.5,0.6],[0.2,0.3],[0.4,0.5]\rangle$ & $\langle[0.5,0.6],[0.7,0.9],[0.3,0.4]\rangle$ \\
$\Theta_{5}$ & $\langle[0.4,0.5],[0.6,0.7],[0.6,0.7]\rangle$ & $\langle[0.6,0.7],[0.3,0.4],[0.3,0.4]\rangle$ & $\langle[0.9,1],[0.4,0.5],[0.3,0.4]\rangle$ & $\langle[0.7,0.8],[0.8,0.9],[0.1,0.2]\rangle$ \\
\hline
\end{tabular}

Table 3

The decision matrix $\widetilde{D M}_{3}$.

\begin{tabular}{lllll}
\hline & $\wp 1$ & $\wp_{2}$ & $\wp_{3}$ & $\wp_{4}$ \\
\hline$\Theta_{1}$ & $\langle[0.7,0.8],[0.4,0.5],[0.4,0.5]\rangle$ & $\langle[0.7,0.8],[0.3,0.4],[0.6,0.7]\rangle$ & $\langle[0.6,0.7],[0.3,0.4],[0.4,0.5]\rangle$ & $\langle[0.5,0.6],[0.4,0.5],[0.4,0.5]\rangle$ \\
$\Theta_{3}$ & $\langle[0.6,0.7],[0.5,0.6],[0.4,0.5]\rangle$ & $\langle[0.7,0.8],[0.6,0.7],[0.5,0.6]\rangle$ & $\langle[0.8,0.9],[0.2,0.3],[0.7,0.8]\rangle$ & $\langle[0.6,0.7],[0.3,0.4],[0.4,0.6]\rangle$ \\
$\Theta_{3}$ & $\langle[0.7,0.8],[0.3,0.4],[0.5,0.6]\rangle$ & $\langle[0.8,0.9],[0.2,0.4],[0.6,0.7]\rangle$ & $\langle[0.8,0.9],[0.2,0.4],[0.4,0.5]\rangle$ & $\langle[0.9,1],[0.1,0.2],[0.5,0.6]\rangle$ \\
$\Theta_{4}$ & $\langle[0.7,0.8],[0.4,0.5],[0.6,0.7]\rangle$ & $\langle[0.6,0.9],[0.1,0.2],[0.7,0.8]\rangle$ & $\langle[0.6,0.7],[0.1,0.2],[0.5,0.6]\rangle$ & $\langle[0.6,0.7],[0.3,0.4],[0.4,0.5]\rangle$ \\
$\Theta_{5}$ & $\langle[0.6,0.7],[0.7,0.8],[0.2,0.3]\rangle$ & $\langle[0.7,0.8],[0.3,0.5],[0.4,0.5]\rangle$ & $\langle[0.7,0.9],[0.3,0.4],[0.4,0.5]\rangle$ & $\langle[0.8,0.9],[0.5,0.6],[0.5,0.6]\rangle$ \\
\hline
\end{tabular}

There are three experts $\widetilde{D M}_{a}(a=1,2,3)$ with importance degrees $(0.25,0.4,0.35)^{T}$, who evaluate the five ETEs with respect to the four attributes with importance degree $(0.15,0.2,0.25,0.4)^{T}$, and provide their information in the form of INNs, which are listed in Tables 1-3.

In the following, we need to select the best alternatives. The precised steps are illustrated as follows:

Step 1. Normalize the decision matrices using equation (28). Since all the attributes are of benefit type so there is no need to normalize it.

Step 2. Determine the supports $\operatorname{Supp}\left(\Re_{i j}^{c}, \Re_{i j}^{d}\right)(i=1,2, \ldots, 5, j=1,2,3,4, c, d=$ $1,2,3, c \neq d$ ) using equation (29). In order to define the supports between $\Re_{i j}^{c}$ and $\Re_{i j}^{d}$, we denote $\left(\operatorname{Supp}\left(\Re_{i j}^{c}, \Re_{i j}^{d}\right)\right)_{5 \times 4}$ as $\operatorname{Supp}{ }^{(c d)}$, which are given as follows:

$$
\text { Supp }^{12}=\text { Supp }^{21}=\left[\begin{array}{cccc}
0.9167 & 0.7 & 0.8 & 0.8333 \\
0.85 & 0.7833 & 0.867 & 0.8667 \\
0.7 & 0.7333 & 0.8333 & 0.7 \\
0.8333 & 0.6 & 0.867 & 0.7833 \\
0.7333 & 0.7333 & 0.8333 & 0.7333
\end{array}\right]
$$




$$
\begin{aligned}
\text { Supp }^{13}=\text { Supp }^{31}= & {\left[\begin{array}{cccc}
0.7833 & 0.7 & 0.7 & 0.8333 \\
0.8833 & 0.75 & 0.6667 & 0.9167 \\
0.7333 & 0.5833 & 0.7167 & 0.7667 \\
0.7333 & 0.7333 & 0.7667 & 0.8 \\
0.7667 & 0.6833 & 0.8167 & 0.7333
\end{array}\right], } \\
\text { Supp }^{23}=\text { Supp }^{3} 2= & {\left[\begin{array}{cccc}
0.8333 & 0.8667 & 0.9 & 0.9333 \\
0.9 & 0.9667 & 0.8 & 0.85 \\
0.7667 & 0.85 & 0.8833 & 0.7333 \\
0.9 & 0.8 & 0.9 & 0.7833 \\
0.7667 & 0.9167 & 0.8833 & 0.7333
\end{array}\right] . }
\end{aligned}
$$

Step 3. Determine the weighted supports $T\left(\Re_{i j}^{c}\right.$ ) of INN $\Re_{i j}^{c}$ by other INNs $\Re_{i j}^{d}(d=$ $1,2,3$ and $c \neq d$ ) by utilizing equation (30), and determine the weight $\varpi_{i j}^{(c)}(i=$ $1,2,3,4,5, j=1,2,3,4, c=1,2,3)$ of INN $\Re_{i j}^{c}(i=1,2,3,4,5, j=1,2,3,4, c=$ $1,2,3)$ by utilizing equation (31). In order to represent $\left(T\left(\Re_{i j}^{c}\right)\right)_{5 \times 4}$ as $\widetilde{T}_{c}(c=1,2,3)$ and $\left(\varpi_{i j}^{(c)}\right)_{5 \times 4}$ as $U_{c}(c=1,2,3)$, which are given as follows:

$$
\begin{aligned}
\widetilde{T}_{1} & =\left[\begin{array}{cccc}
0.6408 & 0.525 & 0.565 & 0.625 \\
0.6492 & 0.5758 & 0.58 & 0.6675 \\
0.5367 & 0.4975 & 0.5842 & 0.5483 \\
0.59 & 0.4967 & 0.615 & 0.5933 \\
0.5617 & 0.5325 & 0.6192 & 0.55
\end{array}\right] ; \\
\widetilde{T}_{2} & =\left[\begin{array}{cccc}
0.5208 & 0.4783 & 0.515 & 0.535 \\
0.5275 & 0.5342 & 0.4967 & 0.5142 \\
0.4433 & 0.4808 & 0.5175 & 0.4317 \\
0.5233 & 0.43 & 0.5317 & 0.47 \\
0.4517 & 0.5042 & 0.5175 & 0.44
\end{array}\right] ; \\
\widetilde{T}_{3} & =\left[\begin{array}{cccc}
0.5292 & 0.5217 & 0.535 & 0.5817 \\
0.5808 & 0.5742 & 0.4867 & 0.5692 \\
0.49 & 0.4858 & 0.5325 & 0.485 \\
0.5433 & 0.5033 & 0.5517 & 0.5133 \\
0.4983 & 0.5375 & 0.5575 & 0.4767
\end{array}\right] ; \\
U_{1} & =\left[\begin{array}{cccc}
0.2640 & 0.2533 & 0.2550 & 0.2581 \\
0.26151 & 0.2528 & 0.2609 & 0.2652 \\
0.2591 & 0.2518 & 0.2573 & 0.2616 \\
0.2570 & 0.2541 & 0.2589 & 0.2628 \\
0.2611 & 0.2516 & 0.2600 & 0.2618
\end{array}\right] ;
\end{aligned}
$$


Table 4

The overall decision matrix $\widetilde{C D M}$.

\begin{tabular}{|c|c|c|c|c|c|c|}
\hline \multicolumn{5}{|l|}{$\wp_{1}$} & \multicolumn{2}{|l|}{$\wp_{2}$} \\
\hline \multicolumn{5}{|c|}{$\begin{array}{l}\langle[0.4482,0.5903],[0.5066,0.6434],[0.3399,0.4733]\rangle \\
\langle[0.5608,0.7529],[0.5077,0.6470],[0.3039,0.4397]\rangle \\
\langle[0.6234,0.7285],[0.5705,0.6868],[0.3717,0.4765]\rangle \\
\langle[0.6264,0.7261],[0.3048,0.4071],[0.4087,0.5227]\rangle \\
\langle[0.4574,0.5579],[0.5225,0.6354],[0.3428,0.4531]\rangle\end{array}$} & \multicolumn{2}{|c|}{$\begin{array}{l}\langle[0.5577,0.6593],[0.3391,0.4442],[0.4088,0.5230]\rangle \\
\langle[0.6251,0.7255],[0.5132,0.6455], \leqslant f t[0.3726,0.4773]\rangle \\
\langle[0.5913,0.7040],[0.4143,0.5437],[0.4088,0.5231]\rangle \\
\langle[0.5848,0.7756],[0.2294,0.3443],[0.5230,0.6361]\rangle \\
\langle[0.4810,0.5912],[0.4091,0.5453],[0.3055,0.4078]\rangle \\
\end{array}$} \\
\hline$U_{2}=$ & $\begin{array}{l}0.3915 \\
0.3875 \\
0.3893 \\
0.3939 \\
0.3883\end{array}$ & $\begin{array}{l}0.3929 \\
0.3937 \\
0.3984 \\
0.3885 \\
0.3951\end{array}$ & $\begin{array}{l}0.3949 \\
0.3954 \\
0.3943 \\
0.3928 \\
0.3898\end{array}$ & $\begin{array}{l}0.38 \\
0.38 \\
0.38 \\
0.38 \\
0.38\end{array}$ & \multicolumn{2}{|c|}{$\left.\begin{array}{l}1 \\
3 \\
1 \\
9 \\
1\end{array}\right]$} \\
\hline$U_{3}=$ & $\begin{array}{l}0.3445 \\
0.3509 \\
0.3517 \\
0.3492 \\
0.3507\end{array}$ & $\begin{array}{l}0.3538 \\
0.3535 \\
0.3498 \\
0.3574 \\
0.3534\end{array}$ & $\begin{array}{l}0.3501 \\
0.3437 \\
0.3484 \\
0.3482 \\
0.3501\end{array}$ & $\begin{array}{l}0.35 \\
0.3 \\
0.35 \\
0.3 \\
0.3\end{array}$ & & . \\
\hline
\end{tabular}

Step 4. Utilize the WINPHM operator (equation (32)) to get the overall decision matrix (and assume that $k=2$ ), which are given in Table 4 .

Step 5. Determine the supports $\operatorname{Supp}\left(\Re_{i j}, \Re_{i q}\right)(i=1,2, \ldots, 5, j=1,2, \ldots, 4 ; q=$ $1,2, \ldots, 4)$ by using equation (33). For simplicity, $\operatorname{Supp}\left(\Re_{i j}, \Re_{i q}\right)_{5 \times 1}$ is denoted by Supp $_{j q}$ to define the supports among the $j$ th and $q$ th column of $\widetilde{C D M}$.

$$
\begin{aligned}
& \text { Supp }_{12}=\text { Supp }_{12}=\left[\begin{array}{l}
0.8894 \\
0.9659 \\
0.9267 \\
0.9239 \\
0.9428
\end{array}\right], \quad \text { Supp }_{13}=\text { Supp }_{31}=\left[\begin{array}{l}
0.8883 \\
0.8752 \\
0.8273 \\
0.8857 \\
0.8487
\end{array}\right], \\
& \text { Supp }_{14}=\text { Supp }_{41}=\left[\begin{array}{l}
0.9475 \\
0.9206 \\
0.8456 \\
0.8352 \\
0.9013
\end{array}\right], \quad \text { Supp }_{23}=\text { Supp }_{32}=\left[\begin{array}{l}
0.8909 \\
0.8969 \\
0.8862 \\
0.8582 \\
0.9016
\end{array}\right], \\
& \text { Supp }_{24}=\text { Supp }_{42}=\left[\begin{array}{l}
0.9093 \\
0.8720 \\
0.7729 \\
0.8811
\end{array}\right], \quad \text { Supp }_{34}=\text { Supp }_{43}=\left[\begin{array}{l}
0.9384 \\
0.9534 \\
0.9200 \\
0.8611 \\
0.8614
\end{array}\right] .
\end{aligned}
$$


Step 6. Determine the weighted supports $T\left(\Re_{i j}\right)$ of INN $\Re_{i j}$ by utilizing equation (34) and determine the weights $\phi_{i j}(j=1,2,3,4)$ of the INNs $\Re_{i j}$ by utilizing equation (35). For computational clarity, we denote $\left(T\left(\Re_{i j}\right)\right)_{5 \times 4}$ as $\widetilde{T}$ and $\left(\phi_{i j}\right)_{5 \times 4}$ as $\widetilde{U}$, which are given as follows:

$$
\begin{aligned}
& \widetilde{T}=\left[\begin{array}{llll}
0.7790 & 0.7147 & 0.6868 & 0.5560 \\
0.7802 & 0.7328 & 0.6920 & 0.5583 \\
0.7304 & 0.7094 & 0.6693 & 0.5312 \\
0.7403 & 0.6623 & 0.6489 & 0.4951 \\
0.7613 & 0.7193 & 0.6522 & 0.5268
\end{array}\right], \\
& \widetilde{U}=\left[\begin{array}{llll}
0.1613 & 0.2074 & 0.2550 & 0.3763 \\
0.1609 & 0.2088 & 0.2548 & 0.3755 \\
0.1591 & 0.2096 & 0.2558 & 0.3755 \\
0.1628 & 0.2073 & 0.2570 & 0.3729 \\
0.1619 & 0.2107 & 0.2531 & 0.3743
\end{array}\right] .
\end{aligned}
$$

Step 7. Using the WINPHM operator in equation (36), to aggregate all the execution values $\Re_{i j}(j=1,2,3,4)$ in the $i$ th line of $\widetilde{C D M}$ and get the comprehensive execution values $\Theta_{i}(i=1,2, \ldots, 5)$ (assume that $\left.k=2\right)$.

$$
\begin{aligned}
& \Theta_{1}=\langle[0.4401,0.5531],[0.4305,0.5385],[0.3498,0.4614]\rangle ; \\
& \Theta_{2}=\langle[0.6068,0.7359],[0.4345,0.5587],[0.3712,0.4904]\rangle ; \\
& \Theta_{3}=\langle[0.6045,0.7137],[0.3484,0.4767],[0.3774,0.4805]\rangle ; \\
& \Theta_{4}=\langle[0.5221,0.6468],[0.3153,0.4443],[0.4129,0.5198]\rangle ; \\
& \Theta_{5}=\langle[0.5221,0.6468],[0.5101,0.6262],[0.3373,0.4390]\rangle
\end{aligned}
$$

Step 8. Determine the score values of $\Theta_{i}(i=1,2,3,4,5)$ by using Definition 5 , we have

$$
\begin{array}{rlrl}
\widetilde{S C}\left(\Theta_{1}\right) & =1.6065, & \widetilde{S C}\left(\Theta_{2}\right)=1.7439, & \widetilde{S C}\left(\Theta_{3}\right)=1.8176, \\
\widetilde{S C}\left(\Theta_{4}\right)=1.7383, & \widetilde{S C}\left(\Theta_{5}\right)=1.6281 .
\end{array}
$$

Then the alternatives can be arranged in decreasing order according to their score values:

$$
\Theta_{3}>\Theta_{2}>\Theta_{4}>\Theta_{5}>\Theta_{1}
$$

Step 9. Based on Definition 6, and the best ETEs is $\Theta_{3}$ while the worst one is $\Theta_{1}$.

\subsection{Effect of the Parameter $k$}

In this subsection, we take different values of the parameter $k$ in the WINPHM operator to observe the ranking results, hence we can determine the score values produced for different values of the parameter $k$, and the ranking results are given in Table 5. 
Table 5

The overall decision matrix $\widetilde{C D M}$.

\begin{tabular}{lll}
\hline & $\wp 3$ & $\wp 4$ \\
\hline$\Theta_{1}$ & $\langle[0.3662,0.4810],[0.3740,0.4734],[0.2646,0.3724]\rangle$ & $\langle[0.4553,0.5563],[0.4397,0.5387],[0.3044,0.4067]\rangle$ \\
$\Theta_{2}$ & $\langle[0.6603,0.7947],[0.3067,0.4417],[0.4156,0.5294]\rangle$ & $\langle[0.6553,0.7538],[0.3382,0.4386],[0.3034,0.4425]\rangle$ \\
$\Theta_{3}$ & $\langle[0.5914,0.7042],[0.1392,0.2745],[0.3048,0.4070]\rangle$ & $\langle[0.7040,0.8332],[0.2277,0.3427],[0.3462,0.4475]\rangle$ \\
$\Theta_{4}$ & $\langle[0.4928,0.5928],[0.1731,0.2735],[0.3336,0.4440]\rangle$ & $\langle[0.4567,0.5586],[0.4874,0.6544],[0.3038,0.4061]\rangle$ \\
$\Theta_{5}$ & $\langle[0.6863,0.8395],[0.3731,0.4727],[0.3042,0.4065]\rangle$ & $\langle[0.5914,0.7041],[0.6535,0.7623],[0.3205,0.4214]\rangle$ \\
\hline
\end{tabular}

Table 6

Scores and ranking of the alternatives for different parameter values.

\begin{tabular}{lll}
\hline$k$ & Score values & Ranking order \\
\hline$k=1$ & $\widetilde{S C}\left(\Theta_{1}\right)=1.7512, \widetilde{S C}\left(\Theta_{2}\right)=1.9127, \widetilde{S C}\left(\Theta_{3}\right)=2.1819$, & $\Theta_{3}>\Theta_{5}>\Theta_{2}>\Theta_{4}>\Theta_{1}$ \\
& $\widetilde{S C}\left(\Theta_{4}\right)=1.9061, \widetilde{S C}\left(\Theta_{5}\right)=1.9670$. & \\
$k=2$ & $\widetilde{S C}\left(\Theta_{1}\right)=1.6065, \widetilde{S C}\left(\Theta_{2}\right)=1.7439, \widetilde{S C}\left(\Theta_{3}\right)=1.8176$, & $\Theta_{3}>\Theta_{2}>\Theta_{4}>\Theta_{5}>\Theta_{1}$ \\
& $\widetilde{S C}\left(\Theta_{4}\right)=1.7383, \widetilde{S C}\left(\Theta_{5}\right)=1.6281$. & \\
$k=3$ & $\widetilde{S C}\left(\Theta_{1}\right)=2.9781, \widetilde{S C}\left(\Theta_{2}\right)=2.9816, \widetilde{S C}\left(\Theta_{3}\right)=2.9869$, & $\Theta_{3}>\Theta_{4}>\Theta_{2}>\Theta_{1}>\Theta_{5}$ \\
& $\widetilde{S C}\left(\Theta_{4}\right)=2.9854, \widetilde{S C}\left(\Theta_{5}\right)=2.9773$. & \\
\hline
\end{tabular}

From Table 5, we can see that when the value of the parameter $k=1$, the ranking order is slightly different, but the best and the worst alternative remain the same as for the parameter value $k=2$. When the value of the parameter $k=3$, then the ranking order is different from the ones obtained for the parameter value $k=1,2$. The best choice remains the same, but the worst alternative is changed. That is, for $k=1,2$ the worst alternative is $\Theta_{1}$, while for $k=3$ the worst alternative is $\Theta_{5}$. These results are reasonable, as we can consider the interrelationship for different number of attributes; when $k=1$, we don't consider the interrelationship of the attributes; when $k=2$, we can take into account the interrelationship between any two attributes; and when $k=3$, we consider the interrelationship among any three attributes. These results show that the proposed AO is more flexible and practical.

\subsection{Comparison with Other Approaches}

In the following, we will utilize the other two approaches to solve the same example, and compare and examine the decision results obtained by these methods. The first approach is based on INBM operator proposed by Ji et al. (2018a), and the second approach is based on INPWA operator proposed by Liu and Tang (2016). The score values and ranking order on these different approaches are shown in Table 6.

From Table 6, we can observe that when the value of the parameter gets $k=1$, there are the same ranking results of our method in this paper with the method in Liu and Tang (2016), while when the value of the parameter gets $k=2$, we get the same ranking results of our method in this paper with the method defined by Ji et al. (2018a). However, they are different in ranking result from the method Liu and Tang. We think these results are reasonable and can explain them as follows.

1. When $k=1$, the method proposed in this paper can reduce into PA operator for INNs, and it is similar to method in Liu and Tang (2016), so these two methods 
Table 7

Score values and ranking order of different approaches.

\begin{tabular}{lll}
\hline Approach & Score values of & Ranking order \\
\hline $\begin{array}{l}\text { Based on INWBM } \\
\text { operator } p=q=1 \text { by }\end{array}$ & $\widetilde{S C}\left(\Theta_{1}\right)=0.2004, \widetilde{S C}\left(\Theta_{2}\right)=0.2326$, & $\Theta_{3}>\Theta_{2}>\Theta_{4}>\Theta_{5}>\Theta_{1}$ \\
Ji et al. $(2018 \mathrm{a})$ & $\widetilde{S C}\left(\Theta_{3}\right)=0.2633, \widetilde{S C}\left(\Theta_{4}\right)=0.2205$ & \\
Based on INWPA & $\widetilde{S C}\left(\Theta_{1}\right)=1.7623, \widetilde{S C}\left(\Theta_{2}\right)=1.9254$, & $\Theta_{3}>\Theta_{5}>\Theta_{2}>\Theta_{4}>\Theta_{1}$ \\
operator $\lambda=1$ by Liu & $\widetilde{S C}\left(\Theta_{3}\right)=2.1943, \widetilde{S C}\left(\Theta_{4}\right)=1.8964$, & \\
and Tang $(2016)$ & $\widetilde{S C}\left(\Theta_{5}\right)=1.9794$ & $\Theta_{3}>\Theta_{2}>\Theta_{4}>\Theta_{5}>\Theta_{1}$ \\
Based on the & $\widetilde{S C}\left(\Theta_{1}\right)=1.6065, \widetilde{S C}\left(\Theta_{2}\right)=1.7439$, & \\
proposed operator in & $\widetilde{S C}\left(\Theta_{3}\right)=1.8176, \widetilde{S C}\left(\Theta_{4}\right)=1.7383$, & \\
this article $(k=2)$ & $\widetilde{S C}\left(\Theta_{5}\right)=1.6281$ & \\
Based on the & $\widetilde{S C}\left(\Theta_{1}\right)=1.7512, \widetilde{S C}\left(\Theta_{2}\right)=1.9127$, & \\
proposed operator in & $\widetilde{S C}\left(\Theta_{3}\right)=2.1819, \widetilde{S C}\left(\Theta_{4}\right)=1.9061$, & \\
this article $(k=1)$ & $\widetilde{S C}\left(\Theta_{5}\right)=1.9670$ & \\
\hline
\end{tabular}

produced the same ranking results. Obviously, this can explain the validity of our proposed method.

2. When $k=2$, the method proposed in this paper can reduce into BM operator for INNs, and it is similar to method in Ji et al. (2018b), so these two methods produced the same ranking results. Obviously, this can further explain the validity of our proposed method.

3. There are different ranking results of our method when $k=1$ and of the method in Liu and Tang (2016) compared to our method when $k=2$ and the method in Ji et al. (2018a), and the reason is that our method when $k=1$ and the method in Liu and Tang (2016) cannot consider the interrelationship of the attributes, while our method when $k=2$ and the method in Ji et al. (2018b) can do it.

Further, we can compare the existing two methods Ji et al. (2018a) and Liu and Tang (2016) with our method in this paper as follows.

(1) Ji et al. (2018a) developed the method based on INWBM operator, and the developed aggregation operators only consider the interrelationship between two attributes and cannot eliminate the effect of awkward data. While the proposed aggregation operator has the properties that it can consider the interrelationship among more than two attributes $(k \geqslant 2)$ or doesn't consider the interrelationship of the attributes (when $k=1$ ), and also remove the effect of awkward data. Obviously, our method is more flexible and practical than the method in Ji et al. (2018b).

(2) Liu and Tang (2016) developed the method based on INPWA operator. The developed operator can only eliminate the effect of awkward data given by the DMs and cannot consider the correlation among attributes. Obviously, our method is also more flexible and practical then the method in Liu and Tang (2016).

In practical MAGDM or MADM problems, our proposed approach is superior to the existing two approaches. The developed aggregation operators in this article have some advantages over the existing aggregation operators which are listed as follows: 
(1) INS is a better mathematical tool to deal with uncertain and vague information than IFS, IVIFS, SVNS.

(2) The developed aggregation operators have the capacity of removing influence of awkward data by PA operator and considering the interrelationship between any number of input arguments at the same time, while some existing aggregation operator can consider interrelationship among two input arguments or remove the effect of awkward data.

(3) The other advantage of the developed aggregation operators is that some existing aggregation operators are special cases of these aggregation operators.

\section{Conclusion}

The HM operator is an aggregation tool that can consider the interrelationship between multiple input parameters, and the PA operator has the property that it can reduce the potency of awkward assessment values in the decision consequences. The INSs are a more powerful tool to handle uncertain information that exists in real life problems. Therefore, for some complex decision-making situations in this article, we combine the conventional HM operator to the traditional PA operator in interval neutrosophic settings and present the two novel interval neutrosophic aggregation operators, that is, the interval neutrosophic power Hamy mean (INPHM) operator and the weighted interval neutrosophic power Hamy mean (WINPHM) operators. Then, some preferable properties and special cases of the developed aggregation operators are discussed. Moreover, based on these developed aggregation operators, we propose a new method to MAGDM. Lastly, the developed approach is applied to some practical problems and shows that the proposed aggregation operators are better and more flexible then some existing aggregation operators. The other feature of the developed aggregation operator is generalization of some existing aggregation operators.

In future, we shall extend the proposed aggregation operator to some other fuzzy information such as Pythagorean fuzzy sets, picture fuzzy sets, linguistic neutrosophic sets, uncertain linguistic sets, unbalance fuzzy linguistic information and apply them to social networking, large-scale group decision making.

Acknowledgements. This paper is supported by the National Natural Science Foundation of China (Nos. 71771140 and 71471172 ), the Special Funds of Taishan Scholars Project of Shandong Province (No. ts201511045).

\section{References}

Atanassov, K.T. (1986). Intuitionistic fuzzy sets. Fuzzy Sets and Systems, (20)(1), 87-96.

Bausys, R., Zavadskas, K.E., Kaklauskas, A. (2015). Application of neutrosophic set to multicriteria decision making by COPRAS. Journal of Economic Computation and Economic Cybernetics Studies and Research, 49, 84-98. 
Cabrerizo, F.J., Al-Hmouz, R., Morfeq, A., Balamash, A.S., Martinez, M.A., Herrera-Viedma, E. (2017). Soft consensus measures in group decision making using unbalanced fuzzy linguistic information. Soft Computing, 21(11), 3037-3050.

Capuano, N., Chiclana, F., Fujita, H., Herrera-Viedma, E., Loia, V. (2018). Fuzzy group decision making with incomplete information guided by social influence. IEEE Transactions on Fuzzy Systems, 26(3), 1704-1718.

Dong, Y., Zhang, H., Herrera-Viedma, E. (2016). Integrating experts' weights generated dynamically into the consensus reaching process and its applications in managing non-cooperative behaviors. Decision Support Systems, (84), 1-15.

Dong, Y., Zhao, S., Zhang, H., Chiclana, F., Herrera-Viedma, E. (2018). A self-management mechanism for non-cooperative behaviors in large-scale group consensus reaching processes. IEEE Transactions on Fuzzy Systems, (26)(6), 3276-3288.

Feng, J., Li, M., Li, Y. (2018). Study of decision framework of shopping mall photovoltaic plan selection based on DEMATEL and ELECTRE III with symmetry under neutrosophic set environment. Symmetry, 10(5), 150 .

Garg, H. (2016). An improved score function for ranking neutrosophic sets and its application to decision-making process. International Journal for Uncertainty Quantification, 6(5), 377-385.

Hara, T., Uchiyama, M., Takahasi, S.E. (1998). A refinement of various mean inequalities. Journal of Inequalities and Applications, 2(4), 387-395.

He, Y.D., Chen, H., Zhou, L., Liu, J., Tao, Z. (2013). Generalized interval-valued Atanassovs intuitionistic fuzzy power operators and their application to group decision making. International Journal of Fuzzy Systems, 15(4), 401-411.

He, Y.D., Jin, Y., Chen, H. (2015a). Intuitionistic fuzzy power geometric Bonferroni means and their application to multiple attribute group decision making. International Journal of Uncertainty, Fuzziness and KnowledgeBased Systems, 23(02), 285-315.

He, Y., Wang, Z.G., Chen, H. (2015b). Hesitant fuzzy power Bonferroni means and their application to multiple attribute decision making. IEEE Transactions on Fuzzy Systems, 23(5), 1655-1668.

Huang, Y.H. Wei, W.G., Wei, C. (2017). VIKOR method for interval neutrosophic multiple attribute group decision-making. Information, 8(4), 144.

Ji, P., Cheng, P.F., Zhang, H.Y., Wang, J.Q. (2018a). Interval-valued neutrosophic Bonferroni mean operators and the application in the selection of renewable energy. New Trends in Neutrosophic Theory and Applications, 2 12-39.

Ji, P. Cheng, P. F. Zhang, H. Y. and Wang, J. Q. (2018b). A projection-based TODIM method under multi-valued neutrosophic environments and its application in personnel selection. Neural Computing and Applications, 29(1), 221-234.

Li, Y., Liu, P., Chen, Y. (2016). Some single valued neutrosophic number heronian mean operators and their application in multiple attribute group decision making. Informatica, 27(1), 85-110.

Liu, P. (2017). Multiple attribute group decision making method based on interval-valued intuitionistic fuzzy power Heronian aggregation operators. Computers \& Industrial Engineering, 108, 199-212.

Liu, P., Liu, Y. (2014). An approach to multiple attribute group decision making based on intuitionistic trapezoidal fuzzy power generalized aggregation operator. International Journal of Computational Intelligence Systems, 7(2), 291-304.

Liu, P., Wang, Y. (2014). Multiple attribute decision-making method based on single-valued neutrosophic normalized weighted Bonferroni mean. Neural Computing and Applications, 25(7-8), 2001-2010.

Liu, P., Tang, G. (2016). Some power generalized aggregation operators based on the interval neutrosophic sets and their application to decision making. Journal of Intelligent \& Fuzzy Systems, 30(5), 2517-2528.

Liu, P., Chen, S.M. (2017). Group decision making based on Heronian aggregation operators of intuitionistic fuzzy numbers. IEEE transactions on cybernetics, 47(9), 2514-2530.

Liu, P., Li, H. (2017). Interval-valued intuitionistic fuzzy power Bonferroni aggregation operators and their application to group decision making. Cognitive Computation, 9(4), 494-512.

Liu, P., Gao, H. (2018). Multicriteria decision making based on generalized Maclaurin symmetric means with multi-hesitant fuzzy linguistic information. Symmetry, 10(4), 81.

Liu, P., You, X. (2017). Interval neutrosophic muirhead mean operators and their application in multiple attribute group decision-making. International Journal for Uncertainty Quantification, 7(4), 303-334.

Liu, P., You, X. (2018). Some linguistic neutrosophic Hamy mean operators and their application to multiattribute group decision making. PloS One, 13(3), e0193027. 
Liu, P., Zhang, X. (2018). Some Maclaurin symmetric mean operators for single-valued trapezoidal neutrosophic numbers and their applications to group decision making. International Journal of Fuzzy Systems, 20(1), 45 61.

Liu, Z., Teng, F., Liu, P., Ge, Q. (2018). Interval-valued intuitionistic fuzzy power Maclaurin symmetric mean aggregation operators and their application to multiple attribute group decision-making. International Journal for Uncertainty Quantification, 8(3), 211-232.

Maclaurin, C. (1729). A second letter to Martin Folkes, Esq; concerning the roots of equations, with demonstration of otherrules of algebra. Philosophical Transactions of the Royal Society of London A, 36, 59-96.

Morente-Molinera, J.A., Kou, G., Pang, C., Cabrerizo, F.J., Herrera-Viedma, E. (2019). An automatic procedure to create fuzzy ontologies from users' opinions using sentiment analysis procedures and multi-granular fuzzy linguistic modelling methods. Information Sciences, 476, 222-238.

Nie, R.X., Wang, J.Q., Zhang, H.Y. (2017). Solving solar-wind power station location problem using an extended weighted aggregated sum product assessment (WASPAS) technique with interval neutrosophic sets. Symmetry, 9(7), 106

Peng, J.J., Wang, J.Q., Wang, J., Zhang, H.Y., Chen, X.H. (2014). An outranking approach for multi-criteria decision-making problems with simplified neutrosophic sets. Applied Soft Computing, 25, 336-346.

Peng, J.J., Wang, J.Q., Wang, J., Zhang, H.Y., Chen, X.H. (2016). Simplified neutrosophic sets and their applications in multi-criteria group decision-making problems. International Journal of Systems Science, 47(10), 2342-2358.

Qin, J. (2017). Interval type-2 fuzzy Hamy mean operators and their application in multiple criteria decision making. Granular Computing, 2(4), 249-269.

Qin, J., Liu, X. (2014). An approach to intuitionistic fuzzy multiple attribute decision making based on Maclaurin symmetric mean operators. Journal of Intelligent \& Fuzzy Systems, 27(5), 2177-2190.

Smarandache, F. (1998). Neutrosophy: Neutrosophic Probability, Set, and Logic: Analytic Synthesis \& Synthetic Analysis.

Smarandache, F. (1999). A unifying field in logics: neutrosophic logic. In: Philosophy American Research Press, pp. 1-141.

Stanujkic, D., Zavadskas, E.K., Smarandache, F., Brauers, W.K., Karabasevic, D. (2017). A neutrosophic extension of the MULTIMOORA method. Informatica, 28(1), 181-192.

Urena, R., Kou, G., Dong, Y., Chiclana, F., Herrera-Viedma, E. (2019). A review on trust propagation and opinion dynamics in social networks and group decision making frameworks. Information Sciences, 478, 461-475.

Wang, H., Smarandache, F., Sunderraman, R., Zhang, Y.Q. (2005). Interval neutrosophic sets and logic: theory and applications in computing. Theory and Applications in Computing, 5.

Wang, H., Smarandache, F., Zhang, Y.Q., Sunderraman, R. (2010). Single valued neutrosophic sets. Infinite Study.

Wei, G., Lu, M. (2018). Pythagorean fuzzy maclaurin symmetric mean operators in multiple attribute decision making. International Journal of Intelligent Systems, 33(5), 1043-1070.

Wu, S., Wang, J., Wei, G., Wei, Y. (2018). Research on construction engineering project risk assessment with some 2-tuple linguistic neutrosophic Hamy mean operators. Sustainability, 1)(5), 1-26.

Xu, Z. (2007). Intuitionistic fuzzy aggregation operators. IEEE Transactions on Fuzzy Systems, 15(6), 11791187.

Xu, Z. (2011). Approaches to multiple attribute group decision making based on intuitionistic fuzzy power aggregation operators. Knowledge-Based Systems, 24(6), 749-760.

Xu, Z., Yager, R.R. (2006). Some geometric aggregation operators based on intuitionistic fuzzy sets. International Journal of General Systems, 35(4), 417-433.

Xu, Z., Yager, R.R. (2011). Intuitionistic fuzzy Bonferroni means. IEEE Transactions on Systems, Man, and Cybernetics, Part B (Cybernetics), 41(2), 568-578.

Yager, R.R. (2001). The power average operator. IEEE Transactions on Systems, Man, and Cybernetics-Part A: Systems and Humans, 31(6), 724-731.

Ye, J. (2014). A multicriteria decision-making method using aggregation operators for simplified neutrosophic sets. Journal of Intelligent \& Fuzzy Systems, 26(5), 2459-2466.

Yu, D. (2015). Triangular Atanassov's intuitionistic fuzzy Bonferroni mean and application to supplier selection. Journal of Intelligent \& Fuzzy Systems, 28(6), 2785-2791.

Yu, D., Wu, Y. (2012). Interval-valued intuitionistic fuzzy Heronian mean operators and their application in multi-criteria decision making. African Journal of Business Management, 6(11), 4158-4168.

Zadeh, L.A. (1965). Fuzzy sets. Information and Control, 8(3), 338-353. 
Zhang, H.Y., Wang, J.Q., Chen, X.H. (2014). Interval neutrosophic sets and their application in multicriteria decision making problems. The Scientific World Journal, 1-15.

Zhang, X., Liu, P., Wang, Y. (2015). Multiple attribute group decision making methods based on intuitionistic fuzzy frank power aggregation operators. Journal of Intelligent \& Fuzzy Systems, 29(5), 2235-2246.

Zhang, H., Wang, J., Chen, X. (2016). An outranking approach for multi-criteria decision-making problems with interval-valued neutrosophic sets. Neural Computing and Applications, 27(3), 615-627.

Zavadskas, E.K., Bausys, R., Lazauskas, M. (2015). Sustainable assessment of alternative sites for the construction of a waste incineration plant by applying WASPAS method with single-valued neutrosophic set. Sustainability, 7(12), 15923-15936.

Zavadskas, E.K., Bausys, R., Kaklauskas, A., Ubarte, I., Kuzminske, A., Gudiene, N. (2017). Sustainable market valuation of buildings by the single-valued neutrosophic MAMVA method. Applied Soft Computing, 57, 7487.

P. Liu received the BS and MS degrees in signal and information processing from Southeast University, Nanjing, China, in 1988 and 1991, respectively, and the PhD degree in information management from Beijng Jiaotong University, Beijing, China, in 2010.

$\mathrm{He}$ is currently a professor with the School of Management Science and Engineering, Shandong University of Finance and Economics, Shandong, China. He is an associate editor of the Journal of Intelligent and Fuzzy Systems, a member of the editorial board of the Journal Technological and Economic Development of Economy, and a member of the editorial board of other 12 journals. He has authored or coauthored more than 200 publications. His research interests include aggregation operators, fuzzy logic, fuzzy decision making, and their applications.

Q. Khan is currently a PhD candidate at the Department of Mathematics and Statistics, International Islamic University, Islamabad, Pakistan. He received his MS degree in mathematics from International Islamic University Islamabad, Pakistan, in 2014 under the supervision of Dr. Tahir Mahmood. He has 25 international publications to his credit. His areas of interest are automata theory, decision making and neutrosophc theory.

T. Mahmood is assistant professor of Mathematics at Department of Mathematics and Statistics, International Islamic University, Islamabad, Pakistan. He received his $\mathrm{PhD}$ degree in mathematics from Quaid-i-Azam University, Islamabad, Pakistan in 2012 under the supervision of professor Dr. Muhammad Shabir. His areas of interest are algebraic structures, fuzzy algebraic structures and soft sets. He has more than 65 international publications to his credit and he has also produced 38 MS students. 